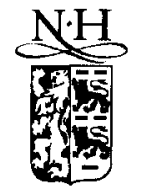

ELSEVIER

\title{
A high-order pathline Godunov scheme for unsteady one-dimensional equilibrium flows
}

\author{
Yun-Hai Tang ${ }^{\mathrm{a}, 1}$, Shih-Tuen Lee ${ }^{\mathrm{a}, 2}$, Jaw-Yen Yang ${ }^{\mathrm{b}, *}$ \\ ${ }^{2}$ Department of Mechanical Engineering, National Taiwan University, Taipei, Taiwan, ROC \\ ${ }^{\mathrm{h}}$ Institute of Applied Mechanics, National Taiwan University, Taipei, Taiwan, ROC
}

Received 12 August 1997

\begin{abstract}
A time-marching Godunov-type method using control cells consisted of particle pathlines and the coordinate lines is described for the computation of unsteady, quasi-one-dimensional equilibrium flows. An unsteady Riemann problem and its solution for equilibrium gases is described and used in the Godunov method. Tannehill's equilibrium air program is used to calculate the equilibrium gas properties. The pathline meshes are automatically solution generated. The use of particle pathline meshes enables a crisp resolution of contact discontinuities and simplifies the Godunov solution procedure. The present particle pathline mesh method shares the same desirable features of Lagrangian description but using an Fulerian description. Extensive computations of various unsteady quasi-one-dimensional equilibrium flows with constant and variable area distribution are included to illustrate the method. Application to a high-temperature shock tube with conical flare indicates that this pathline Godunov method can be useful for estimating the reservoir conditions and the effective time duration for the reflection shock tunnel. (C) 1998 Elsevier Science S.A. All rights reserved.
\end{abstract}

\section{Introduction}

In [1,2], we have described a streamline Godunov-type scheme for the computation of steady, twodimensional and three-dimensional axisymmetrical, supersonic/hypersonic flows for perfect and equilibrium gases. In this work, we shall describe the similar numerical approach extended to the computation of unsteady quasi-one-dimensional perfect and equilibrium flows. In order to implement the Godunov method [3], one has to find the solution for the unsteady Riemann problem for an equilibrium gas. One of the most important tasks one has to deal with in order to obtain accurate and efficient numerical solutions of inviscid equilibrium flows, is to be able to obtain the accurate equilibrium gas properties. Since the equation of state for an equilibrium real gas is not available in closed form, it must either be obtained by solving a set of nonlinear chemical equilibrium equations, or approximated by some curve fit or a table lookup. In recent years, formulations for various approximate Riemann solver for the Euler equations for equilibrium gases have been reported [4-9]. Here, we use the Tannehill's equilibrium air program [10] to calculate the real gas properties. In [10], the equilibrium gas properties such as pressure, temperature and speed of sound, as functions of density and internal energy, can be efficiently calculated. This program is simple and efficient and thus is very suitable for modern computational fluid dynamics, particularly for high speed flows. Other general equilibrium gas laws applied to numerical formulations for various approximate Riemann solvers including the Steger-Warming flux vector splitting [11], the van Leer flux vector splitting [12], and Roe's approximate Riemann solver [13], have been given.

\footnotetext{
* Corresponding author. Professor.

' Graduate Student.

${ }^{2}$ Professor.
} 
The purpose of the present work is to construct an efficient and second-order Godunov-type time marching scheme for unsteady quasi-one-dimensional equilibrium flow computations based on the unsteady Riemann problem and its solution for an equilibrium gas using pathline meshes. As a building block, the Riemann problem and its solution play an essential role in the Godunov-type scheme in the numerical solution of inviscid compressible gas flows. The solution procedure for real gases although is similar to that for the perfect gases, however, due to the different real gas models implemented, the detailed steps can be quite different. Here, Tannehill's equilibrium air model is used, although other equilibrium gas program of Vinokur and Liu [14] can also be used. In this work, pressure is used as the independent thermodynamic variables in order to meet the condition of pressure equality at both sides of contact discontinuity and the real gas properties are calculated due to Srinivasan et al. [10] can also be used.

The merits of using pathline meshes as control cells in the Godunov method are similar to the streamline meshes as described in [1,2], which is motivated by the generalized Lagrangian methods by Loh and Hui [15]. Basically, the use of pathline meshes shares the same desirable features of streamline Godunov method $[1,2]$ via simplifying the Godunov solution procedure and producing crisp resolution of the contact discontinuities since for unsteady flow a contact discontinuity coincides a pathline. To improve the accuracy of the basic Godunov method which is first-order accurate we extend it to second-order method by adopting an essentially nonoscillatory interpolation due to Harten and Osher [16] (see also [1]).

In Section 2, the unsteady quasi-one-dimensional Euler equations of inviscid compressible equilibrium flows are described. In Section 3, the pathline Godunov-type scheme of second-order accuracy is briefly outlined. The elementary waves of the unsteady Riemann problem for an equilibrium gas and its solutions are given in Section 4. Formulations are derived using pressure as independent thermodynamic variable. In Section 5, numerical results for various unsteady quasi-one-dimensional equilibrium flow problems with constant and variable area distribution are given to illustrate the feature of the present method. Some concluding remarks are made in Section 6.

\section{Unsteady Euler equations of real gas}

The Euler equations of motion of an inviscid non-heat-conducting fluid for unsteady quasi-one-dimensional flows in conservation form can be written as

$$
\frac{\partial \boldsymbol{U}}{\partial t}+\frac{\partial \boldsymbol{E}}{\partial x}+\boldsymbol{S}=\mathbf{0}
$$

where

$$
\boldsymbol{U}=\left(\begin{array}{c}
\rho \\
\rho u \\
\rho E
\end{array}\right)=\left(\begin{array}{c}
\mathrm{U}_{1} \\
\mathrm{U}_{2} \\
\mathrm{U}_{3}
\end{array}\right), \quad \boldsymbol{E}=\left(\begin{array}{c}
\rho u \\
\rho u^{2}+P \\
\rho u H
\end{array}\right), \quad S=\frac{\aleph}{A} \frac{\mathrm{d} A}{\mathrm{~d} x}\left(\begin{array}{c}
\rho u \\
\rho u^{2} \\
\rho u H
\end{array}\right) .
$$

Here, $t$ is time, $x$ is $x$-coordinate, $\rho$ is the density of the gas mixture, $u$ is the local velocity, $P$ is the pressure of the gas mixture, $E$ is the total internal energy per unit mass, and $h$ is the total enthalpy per unit mass. The value of $\mathcal{X}$ is 0 for the constant area case and 1 for the variable area one with area distribution $A=A(x)$. The relations among the state variables for equilibrium gases are further defined by two equations of state (EOS) which are taken in the form

$$
P=P(\rho, T), \quad h=h(\rho, T),
$$

where $T$ is the temperature of the mixture, and $h$ is the specific enthalpy. From definition, the total enthalpy per unit mass $H$ is

$$
H=h(\rho, P)+\frac{u^{2}}{2}=e(\rho, P)+\frac{P}{\rho}+\frac{u^{2}}{2} .
$$

Here, $e$ is the specific internal energy. For an inviscid non-heat-conducting flow, $H$ always conserved along the same pathline.

With the state variable vector $\boldsymbol{Q}=(\rho, u, P)^{\mathrm{T}}$, the matrix $[\partial U / \partial Q]^{-1}[\partial E / \partial \boldsymbol{Q}]$ has three real eigenvalues 


$$
\begin{aligned}
& \lambda_{0}=u, \\
& \lambda_{ \pm}=u \pm a,
\end{aligned}
$$

where $a$ is the speed of sound. Since the system (1) is hyperbolic, a marching method in time direction can be constructed to solve Eq. (1). Here, we consider a time-marching method based on the Godunov scheme using a pathline mesh system.

To solve the governing equations with the source terms, like Eq. (1), we can apply the splitting technique. That is, in the case of variable-area flow, the numerical integrations for the source terms can be treated separately by using an appropriate time-marching step. Let $\boldsymbol{U}^{n}$ represent finite difference approximation to the conserved variables $U$ at $t=n \Delta t$, the present computational scheme can be written as

$$
U^{n+1}=\mathscr{L}^{S}(\Delta t / 2) \mathscr{L}^{E}(\Delta t) \mathscr{L}^{S}(\Delta t / 2) U^{n} .
$$

Here, the operator $\mathscr{L}^{S}$ represents the integration of source terms, satisfying

$$
\frac{\partial \boldsymbol{U}}{\partial t}+\boldsymbol{S}=\mathbf{0}
$$

and the operator $\mathscr{L}^{E}$ denotes the finite-volume integration of equation satisfying

$$
\frac{\partial \boldsymbol{U}}{\partial t}+\frac{\partial \boldsymbol{E}}{\partial x}=\mathbf{0} \text {. }
$$

The operator $\mathscr{L}^{S}$ is defined by

$$
\mathscr{L}^{S}(\Delta t) U^{n}=U^{n}-\Delta t^{n} S\left(Q_{i}^{n}\right)+\frac{\left(\Delta t^{n}\right)^{2}}{2}\left(\frac{\partial S}{\partial U}\right)_{i}^{n} S\left(Q_{i}^{n}\right)
$$

The finite-volume formulation of operator $\mathscr{L}^{E}$ will be described in Section 3.

\section{Pathline Godunov method}

In this section, we describe a Godunov-type scheme for solving Eq. (1) using an second-order ENO interpolation with pathline control cells for the unsteady Euler equations in quasi-one-dimensional flows.

\subsection{Finite-volume formulation}

Let the marching direction be in the $t$-coordinate and the mesh is oriented such that $t_{i}^{n}=$ constant $=t^{n}$ for all $i$. Here, $n$ and $i$ refer to the marching step number and cell number, respectively. The marching time-interval, $\Delta t^{n}=t^{n+1}-t^{n}$, is chosen to satisfy the usual Courant-Friedrichs-Lewy (CFL) linear stability condition based on the elementary wave systems of the unsteady Riemann problem at the cell interfaces of all pathline cells. The computational domain in $x-t$ plane is divided into a system of control cells which in the $x$-direction are centered at $x_{i}^{n}$ and have a width of $\Delta x_{i}^{n}=x_{i+1 / 2}^{n}-x_{i-1 / 2}^{n}$. The computational domain is shown in Fig. 1.

Here, we apply the splitting technique i.e. let $S=0$ in the step $\mathscr{L}^{E}(\Delta t)$ for solving the corresponding Riemann problem of Eq. (1): $\partial U / \partial t+\partial E / \partial x=$. Then, the difference equations for the $i$ th zone are obtained by integrating $\partial U / \partial t+\partial E / \partial x=\mathbf{0}$ over the $i$ th cell and applying the divergence theorem. That is

$$
\iint_{D}\left(\frac{\partial \boldsymbol{U}}{\partial t}+\frac{\partial \boldsymbol{E}}{\partial x}\right) \mathrm{d} x \mathrm{~d} t=\int_{\partial D}(\boldsymbol{U}, \boldsymbol{E}) \cdot \overrightarrow{\boldsymbol{n}} \mathrm{d} l
$$

where $D$ is the control cells on the $(x, t)$ plane, $\partial D$ is the boundary of the control cells $D, \overrightarrow{\boldsymbol{n}}$ is the outward unit normal to $\partial D$, and $\mathrm{d} l=\sqrt{\mathrm{d} x^{2}+\mathrm{d} t^{2}}$ is the infinitesimal line segment on $\partial D$ in the $x-t$ plane. Expanding Eq. (10), it can be expressed in terms of numerical fluxes as

$$
U_{i}^{n+1}=U_{i}^{n} \frac{\Delta x_{i}^{n}}{\Delta x_{i}^{n+1}}-\frac{\Delta t^{n}}{\Delta x_{i}^{n+1}}\left(G_{i+1 / 2}^{N}-G_{i-1 / 2}^{N}\right),
$$




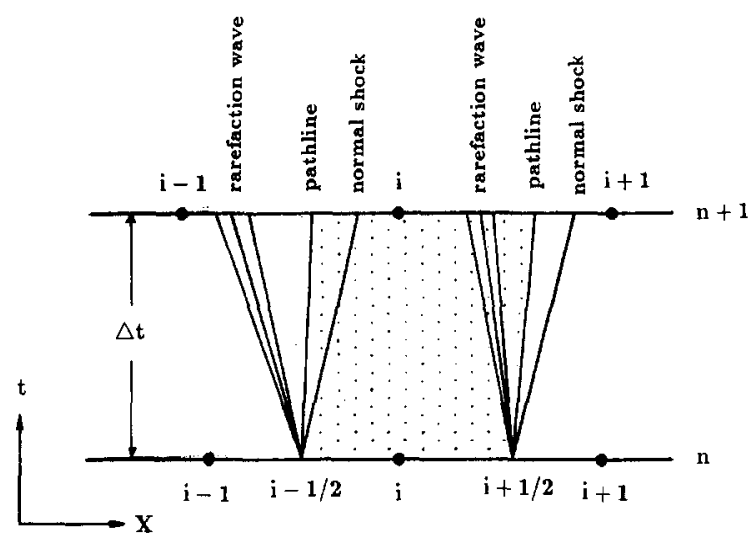

Fig. 1. Pathline meshes in $(x, t)$ coordinates for the streamline Godunov method.

where the numerical flux $G_{i+1 / 2}^{N}$ is defined as

$$
\boldsymbol{G}_{i+1 / 2}^{N}=\boldsymbol{E}_{i+1 / 2}^{n+1 / 2}-s_{i+1 / 2}^{n} \boldsymbol{U}_{i+1 / 2}^{n+1 / 2},
$$

and $s_{i+1 / 2}^{n}$ is the slope of the cell interface at $x_{i+1 / 2}^{n+1 / 2}$, and is given approximately by

$$
s_{i+1 / 2}^{n}=\frac{x_{i+1 / 2}^{n+1}-x_{i+1 / 2}^{n}}{\Delta t^{n}} .
$$

Here, for any quantity $f$, the cell average of $f$ is

$$
f_{i}^{n}=\frac{1}{\Delta x_{i}^{n}} \int_{x_{i-1 / 2}^{n}}^{x_{i+1 / 2}^{n}} f\left(x, t^{n}\right) \mathrm{d} x,
$$

and the time average of $f$ along the right and left boundaries of cells is

$$
f_{i+1 / 2}^{n+1 / 2}=\frac{1}{\Delta t^{n}} \int_{t^{n}}^{t^{n+1}} f\left(x_{i+1 / 2}^{n}+s_{i+1 / 2}^{n} \cdot\left(t-t^{n}\right), t\right) \mathrm{d} t .
$$

In the first-order Godunov scheme, the $i$ th cell average of state variables $\boldsymbol{Q}_{i}^{n}$, at marching step $n$ is considered as constant within that cell and the numerical flux $\boldsymbol{G}_{i+1 / 2}^{N}$ along the interface between the $i$ th cell and the $(i+1)$ th cell from marching step $n$ to $n+1$ is to be obtained from the self-similar solution $\mathrm{R}\left[\left(x-x_{i+1 / 2}^{n}\right) /\left(t-t^{n}\right)\right.$; $\left.Q_{i}^{n}, Q_{i+1}^{n}\right]$ at $x=x_{i+1 / 2}^{n+1 / 2}$ to the unsteady Riemann problem formed by two adjacent piecewise constant flow states $Q_{i}^{n}$ and $Q_{i+1}^{n}$, using the procedure to be described in Section 4.

\subsection{Numerical procedure}

The algorithm for computing the interface flux from the initial data, say step $n$, and updating solutions to step $n+1$ may be divided into a number of steps. There are

Step 1. 'Decode' $\boldsymbol{U}_{i}^{n}$ to obtain $\boldsymbol{Q}_{i}^{n}$ by using the following relations:

$$
\rho=U_{1}, \quad u=\frac{U_{2}}{U_{1}}
$$

and $P$ is determined from the equation state (3) such that

$$
P=P(\rho, e),
$$

where 


$$
e=\frac{U_{3}}{U_{1}}-\frac{U_{2}^{2}}{2 U_{1}^{2}} .
$$

Step 2. Apply a second-order ENO representation to evaluate the state variable pair $\left(\overline{\boldsymbol{Q}}_{i+1 / 2}^{+}, \overline{\boldsymbol{Q}}_{i+1 / 2}^{-}\right)$in the left and right side of cell interfaces. In Eq. (11), the intermediate values $\boldsymbol{U}_{i+1 / 2}^{n+1 / 2}, \boldsymbol{E}_{i+1 / 2}^{n+1 / 2}$ and also $\boldsymbol{G}_{i+1 / 2}^{N}$ can be obtained more accurately at the cell interfaces using a second-order ENO interpolation and are given by

$$
\begin{aligned}
& \boldsymbol{U}_{i+1 / 2}^{n-}=\boldsymbol{U}_{i}^{n}+\frac{1}{2} Z_{i}^{n} \Delta x_{i}^{n}, \\
& \boldsymbol{U}_{i-1 / 2}^{n+}=\boldsymbol{U}_{i}^{n}-\frac{1}{2} Z_{i}^{n} \Delta x_{i}^{n},
\end{aligned}
$$

with

$$
\mathrm{Z}_{i}^{n}=\mathrm{m}\left(a_{i}, b_{i}\right),
$$

where

$$
\begin{aligned}
a_{i} & =\frac{\Delta_{+} U_{i}^{n}}{\Delta_{+} x_{i}^{n}}-\eta \Delta x_{i}^{n} \overline{\mathrm{m}}\left(\frac{\Delta_{+} \Delta_{+} U_{i}^{n}}{\Delta_{+} \Delta_{+} x_{i}^{n}}, \frac{\Delta_{-} \Delta_{+} U_{i}^{n}}{\Delta_{-} \Delta_{+} x_{i}^{n}}\right), \\
b_{i} & =\frac{\Delta_{-} U_{i}^{n}}{\Delta_{-} x_{i}^{n}}-\eta \Delta x_{i}^{n} \overline{\mathrm{m}}\left(\frac{\Delta_{+} \Delta_{-} U_{i}^{n}}{\Delta_{+} \Delta_{-} x_{i}^{n}}, \frac{\Delta_{-} \Delta_{-} U_{i}^{n}}{\Delta_{-} \Delta_{-} x_{i}^{n}}\right) .
\end{aligned}
$$

Here, $\Delta_{ \pm} U_{i}^{n}= \pm\left(U_{i \pm 1}^{n}-U_{i}^{n}\right)$ and $\Delta_{ \pm} x_{i}^{n}= \pm\left(x_{i \pm 1}^{n}-x_{i}^{n}\right)$ denote the usual forward and backward difference operators. In Eq. (21), if $\eta=0$, one has a second-order TVD scheme, and if $\eta=1 / 2$, one has a uniformly second-order ENO scheme. The limiter functions $m$ and $\bar{m}$ are defined, respectively, by

$$
\mathrm{m}(a, b)= \begin{cases}c \min (|a|,|b|), & \text { if } \operatorname{sgn}(a)=\operatorname{sgn}(b)=c, \\ 0, & \text { otherwise },\end{cases}
$$

and

$$
\overline{\mathbf{m}}(a, b)= \begin{cases}a, & \text { if }|a| \leqslant|b|, \\ b, & \text { if }|a|>|b| .\end{cases}
$$

Then, integrate $U_{i \pm 1 / 2}^{n \mp}$ into forward step $n+1 / 2$ such as

$$
\begin{aligned}
& \bar{U}_{i+1 / 2}^{-}=U_{i+1 / 2}^{n-}-\frac{\Delta t^{n}}{2 \Delta x_{i}^{n}}\left[\boldsymbol{E}\left(\boldsymbol{U}_{i+1 / 2}^{n-}\right)-\boldsymbol{E}\left(\boldsymbol{U}_{i-1 / 2}^{n+}\right)\right] \\
& \bar{U}_{i-1 / 2}^{+}=\boldsymbol{U}_{i-1 / 2}^{n+}-\frac{\Delta t^{n}}{2 \Delta x_{i}^{n}}\left[\boldsymbol{E}\left(\boldsymbol{U}_{i+1 / 2}^{n-}\right)-\boldsymbol{E}\left(\boldsymbol{U}_{i-1 / 2}^{n+}\right)\right]
\end{aligned}
$$

where $\boldsymbol{E}\left(\boldsymbol{U}_{i \pm 1 / 2}^{n \mp}\right)$ are the inviscid fluxes $\boldsymbol{E}$ evaluated from the conserved variables $\boldsymbol{U}_{i \pm 1 / 2}^{n \mp}$. After obtaining $\bar{U}_{i \pm 1 / 2}^{\mp}$, we then calculate $\bar{Q}_{j \pm 1 / 2}^{\mp}$ by using the decoding formula (16)-(17).

Step 3. Obtain the self-similar solution $\mathrm{R}\left[\left(x-x_{i+1 / 2}^{n}\right) /\left(t-t_{i+1 / 2}^{n}\right) ; \bar{Q}_{i+1 / 2}^{+}, \bar{Q}_{i+1 / 2}^{-}\right]$by solving the unsteady Riemann problem for equilibrium gases. The solution states along the right and left boundaries of cells, $Q_{i \pm 1 / 2}^{n+1 / 2}$, are used to evaluate the numerical fluxes $G_{i \pm 1 / 2}^{N}$ along $\left(x-x_{i \pm 1 / 2}^{n}\right) /\left(t-t_{i \pm 1 / 2}^{n}\right)=s_{i \pm 1 / 2}^{n}$.

Step 4. Generate the pathline meshes. The pathline meshes are generated by defining

$$
t_{i}^{n+1}=t_{i}^{n}+\Delta t^{n}
$$

and

$$
x_{i \pm 1 / 2}^{n+1}=x_{i \pm 1 / 2}^{n}+\Delta t^{n} s_{i \pm 1 / 2}^{0}=x_{i \pm 1 / 2}^{n}+\Delta t^{n}\left(u^{*}\right)_{i \pm 1 / 2}^{n+1 / 2} .
$$

Here, $\Delta t^{n}$ is the marching time-interval from step $n$ to step $n+1$ and $s_{i \pm 1 / 2}^{0}$ are the slopes of the 0 -waves at the cell interfaces $x=x_{i \pm 1 / 2}^{n+1 / 2}$ which are also equal to the particle velocities at the contact discontinuities, come from 
the unsteady Riemann solutions at the corresponding cell interfaces, i.e. $s_{i \pm 1 / 2}^{0}=\left(u^{*}\right)_{i \pm 1 / 2}^{n+1 / 2}$. From the CFL criterion, $\Delta t^{n}$ can be estimated by

$$
\Delta t^{n}=\min \left[\frac{\Delta x_{i}^{n}}{\left|s_{i-1 / 2}^{+}\right|+\left|\overline{s_{i+1 / 2}^{-}}\right|}\right],
$$

where $s_{i \pm 1 / 2}^{\mp}$ arc the maximum slopes of the,-+ waves at the cell interfaces $x=x_{i \pm 1 / 2}^{n+1 / 2}$, determined from the Riemann solution at the corresponding cell interfaces. Then, we can determine the cell width and the $x$-coordinate of the cell at the marching step $n$ as

$$
\Delta x_{i}^{n+1}=x_{i+1 / 2}^{n+1}-x_{i-1 / 2}^{n+1},
$$

and

$$
x_{i}^{n+1}=\frac{1}{2}\left(x_{i+1 / 2}^{n+1}+x_{i-1 / 2}^{n+1}\right)=x_{i}^{n}+\Delta t^{n} \frac{s_{i+1 / 2}^{0}+s_{i-1 / 2}^{0}}{2} .
$$

Note that the mean slope of pathline for the cell is approximately equal to the average of the slopes at the right and left interfaces of the cell.

Step 5. Finally, the solutions of conserved variables at step $n+1, U_{i}^{n+1}$, can be obtained from Eq. (11), and then calculate the state variables $Q_{i}^{n+1}$ by using the decoding formula (16)-(17).

At this stage the numerical procedures of pathline Godunov method for marching from $n$ to $n+1$ are completed. To march forward further in $t$, one goes back to Step 1 and repeats Steps 1-5.

If a solid endwall is present in the flow, it must be a particle pathline. At this endwall the particle pathline must be matched with the movement history of the solid endwall. So the condition to be imposed on the solid boundary is

$$
\frac{\mathrm{d} x_{w}}{\mathrm{~d} t}=u_{w}
$$

where $x_{w}(t)$ is the movement history of the solid endwall and $u_{w}$ is the moving speed of the solid endwall. This results in a boundary Riemann problem for equilibrium gases and is solved using the procedure described in Section 4.

\section{Unsteady Riemann problem for quasi-1-D flows of equilibrium gases}

The unsteady Riemann problem for quasi-one-dimensional flows for equilibrium gases is specified as the system (1) of hyperbolic conservation laws subject to the initial condition

$$
Q\left(x, t=t_{0}\right)= \begin{cases}Q_{\mathrm{R}}, & x>x_{0}, \\ Q_{\mathrm{L}}, & x \leqslant x_{0},\end{cases}
$$

where $Q=(\rho, u, P)^{\mathrm{T}}$ and subject to the equation of state

$$
P=P(\rho, e),
$$

where the flow states are given at the initial data line $t=t_{0}$ and $Q_{\mathrm{R}}$ and $Q_{\mathrm{L}}$ denote the right and left states, respectively. The equation of state is prescribed as a given function of any two thermodynamic variables, such as $\rho$ and $e$.

The solution to the above unsteady Riemann problem for equilibrium gases is self-similar in the variable $\left(x-x_{0}\right) /\left(t-t_{0}\right)$ and consists of three types of elementary wave $+, 0,-$, namely, the moving normal shock wave, the contact discontinuity, and the centered rarefaction wave, in the order from left to right, as shown in Fig. 2. The \pm -waves may be either normal shocks or rarefaction waves while 0 -wave is always a contact discontinuity. In order to construct these waves, one needs to determine the intersection $\left(P^{*}, u^{*}\right)$ in the $P-u$ plane, as shown in Fig. 3. It is noted that although the classification of the elementary waves for equilibrium gases is the same as that for perfect gases, nevertheless, the real gas effects complicate the formula considerably and exact formula cannot be found explicitly and numerical solution has to be pursued. Denote this Riemann 

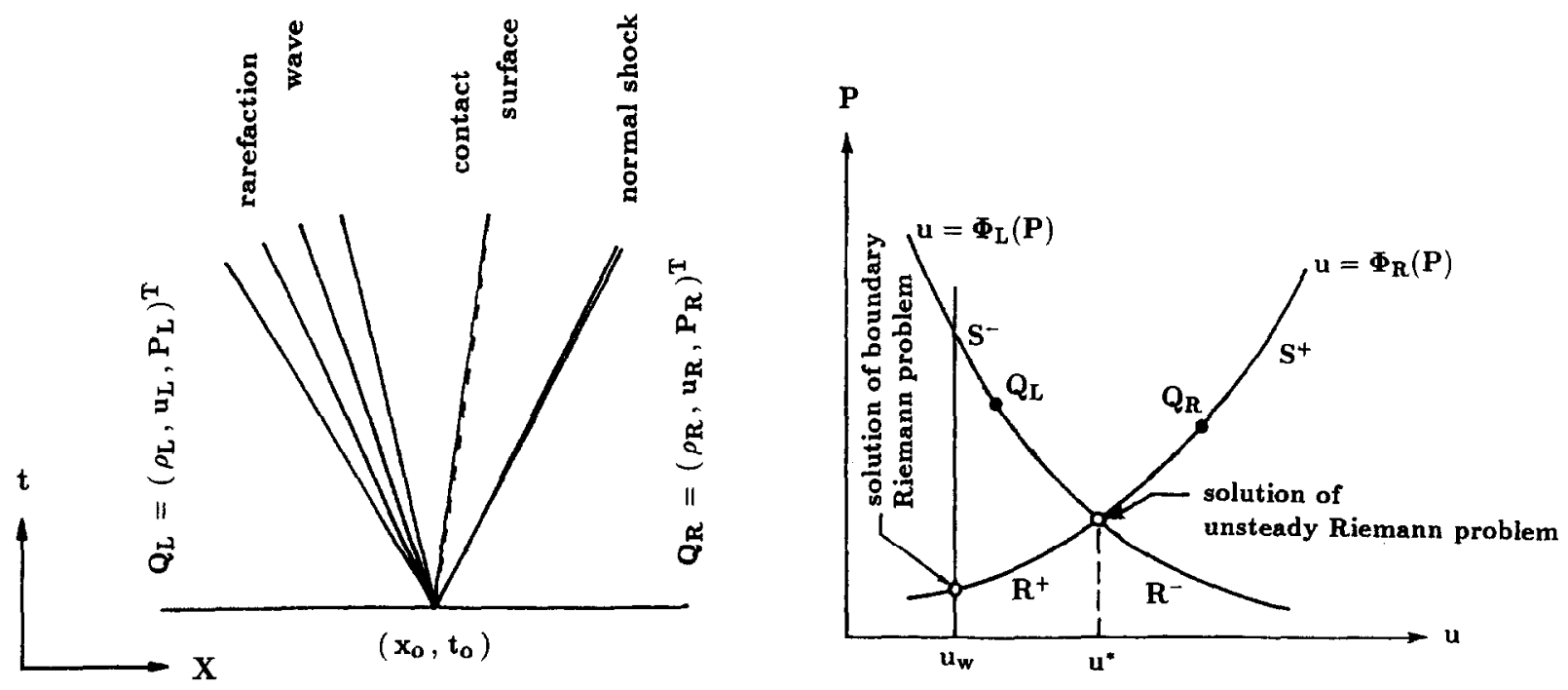

Fig. 2. Elementary wave system in the solution of unsteady Riemann problem.

Fig. 3. Solution for the unsteady Riemann problem in the $(P, u)$ plane.

solution as $\mathrm{R}\left[\left(x-x_{0}\right) /\left(t-t_{0}\right) ; Q_{\mathrm{L}}, Q_{\mathrm{R}}\right]$. These elementary waves can be used to construct a solution to the unsteady Riemann problem for quasi-one-dimensional flows.

\subsection{Elementary wave systems for equilibrium gases}

Let $Q_{0}$ and $Q$ be states ahead of and behind any one of the elementary waves, then there are three cases:

(A) The wave is a contact discontinuity $\left(P=P_{0}\right)$

In this case, from the physical feature of the contact surface, we have

$$
P(\rho, e)=P_{0}\left(\rho_{0}, e_{0}\right)=P^{*}
$$

and

$$
u=u_{0}=u^{*} .
$$

That is, the pressure $P$ and particle velocity $u$ must be identical on either side of contact discontinuity, which holds for perfect and equilibrium gases. The density $\rho$, internal energy $e$, and the other thermodynamic variables may jump abruptly.

(B) The wave is a moving normal shock wave $\left(P>P_{0}\right)$

In this case, from the moving normal shock relations and the equation of state, we have the increment of local velocity, $\delta u$, as

$$
\delta u= \pm\left(1-\frac{\rho_{0}}{\rho}\right) \sqrt{\frac{P-P_{0}}{\rho-\rho_{0}} \frac{\rho}{\rho_{0}}},
$$

for the right- and left-moving normal shock waves, respectively.

The changes of the thermodynamic variables across a moving normal shock wave can be expressed by the Rankine-Hugoniot relation,

$$
e-e_{0}=\frac{P+P_{0}}{2}\left(\frac{1}{\rho_{0}}-\frac{1}{\rho}\right)
$$




$$
\frac{\rho_{0}}{\rho}=1-2 \rho_{0} \frac{e(P, \rho)-e_{0}}{P+P_{0}}
$$

This Rankine-Hugoniot jump condition is an implicit relation between pressure $P$ and density $\rho$. If $P$ is known, $\rho$ can be obtained by defining the object function

$$
\tilde{f}(\rho)=\frac{\rho_{0}}{\rho}+2 \rho_{0} \frac{e(\rho, e)-e_{0}}{P+P_{0}}-1
$$

and using an appropriate numerical procedure.

Having the flow properties ahead of and behind the moving normal shock wave, we can obtain $\delta u$ from Eq. (34). The local flow velocity $u$ is then

(a)

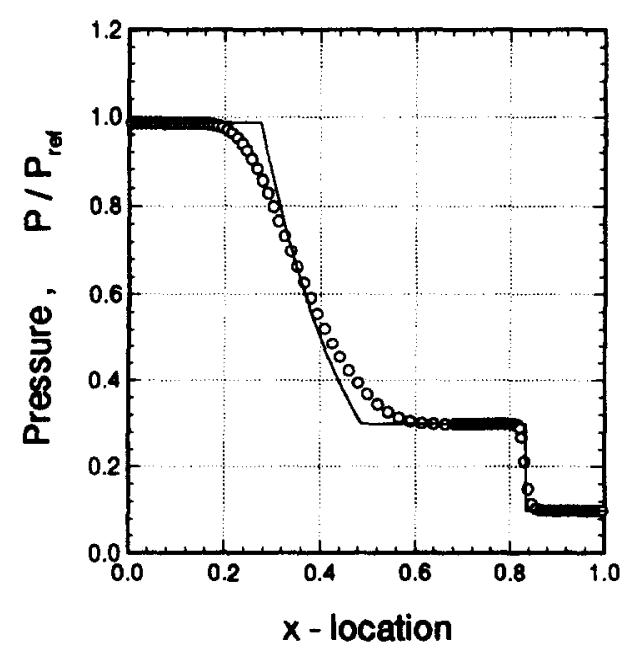

(c)

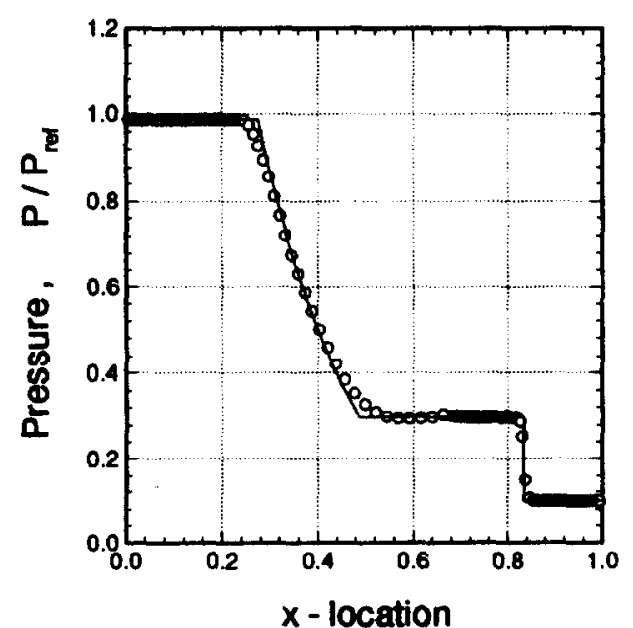

(b)

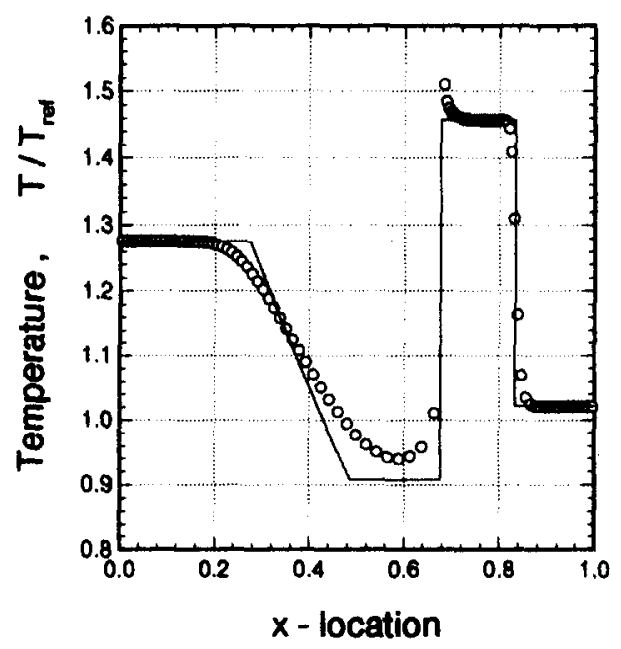

(d)

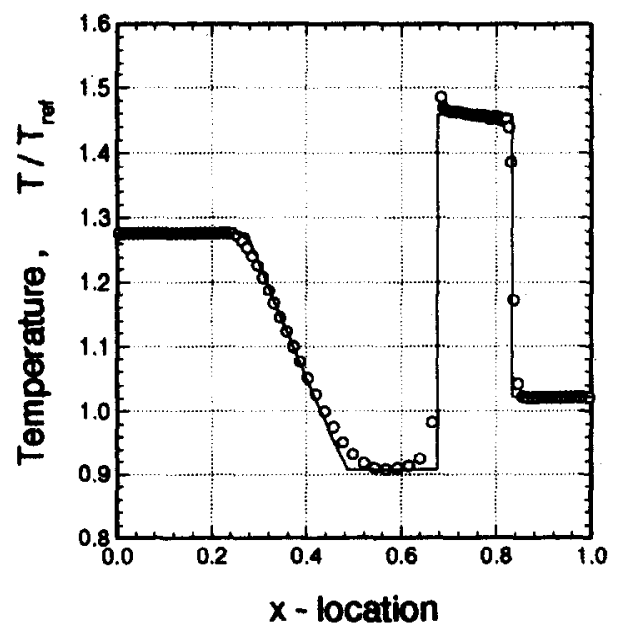

Fig. 4. Solution of Sod's first shock tube problem for a perfect air at time $t=0.6 \mathrm{~ms}$. A comparison of numerical results (circles) and exact solution (solid-line). (a) and (b) First-order Godunov scheme; (c) and (d) second-order ENO scheme. 
$\begin{cases}u=\Phi_{\mathrm{R}}(P)=u_{0}+\delta u & \text { (for the flow on the right) }, \\ u=\Phi_{\mathrm{L}}(P)=u_{0}-\delta u & \text { (for the flow on the left). }\end{cases}$

(C) The wave is a centered rarefaction wave $\left(P<P_{0}\right)$

In this case, from the theory of finite wave, we have the compatibility equations $\mathrm{d} u \pm \frac{\mathrm{d} P}{\rho a}=0 \quad$ along characteristic lines $\mathrm{d} x / \mathrm{d} t=u \pm a$

or

$u \pm \int \frac{\mathrm{d} P}{\rho a}=$ const.

(a)

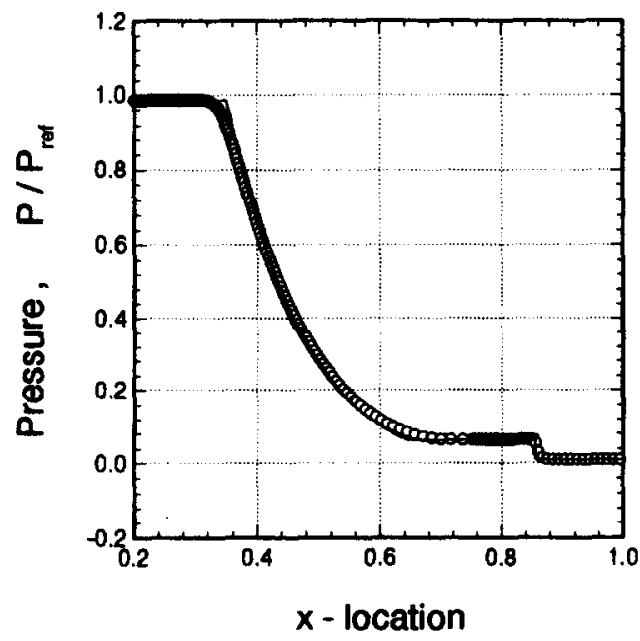

(c)

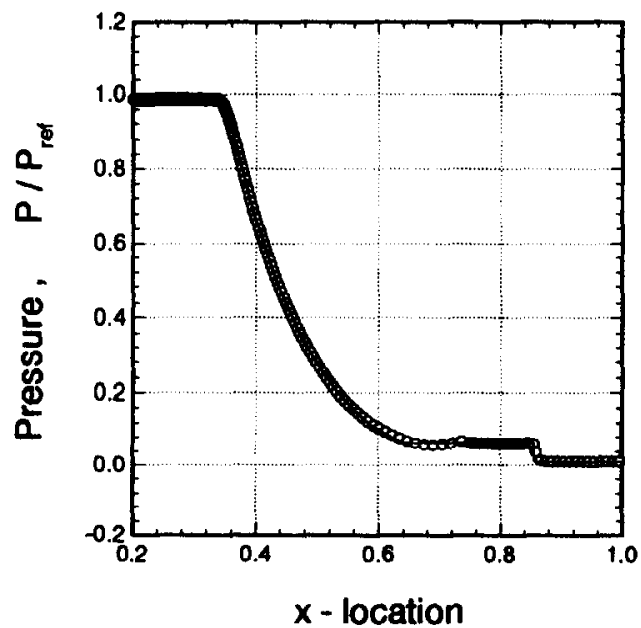

(b)

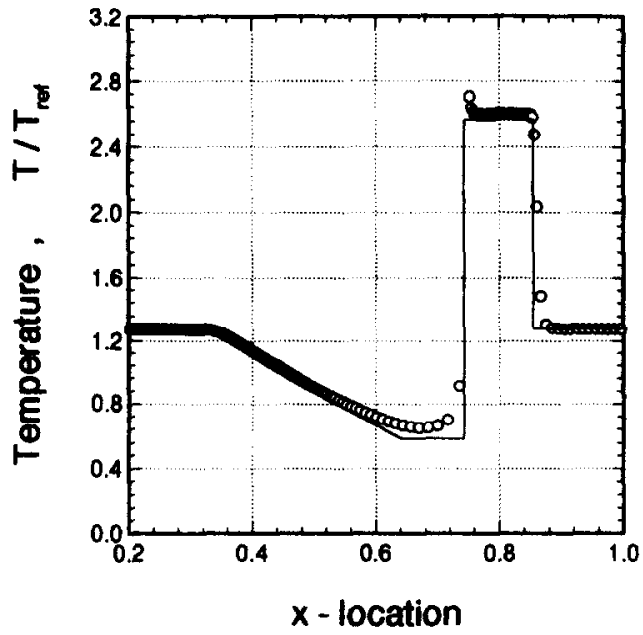

(d)

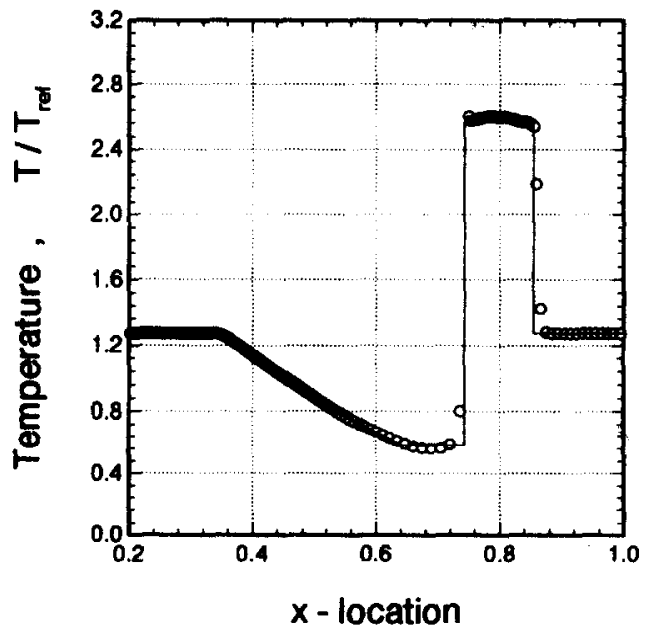

Fig. 5. Solution of Sod's second shock tube problem for a perfect air at time $t=0.4 \mathrm{~ms}$. A comparison of numerical results (circles) and exact solution (solid-line). (a) and (b) First-order Godunov scheme; (c) and (d) second-order ENO scheme. 
hold through the left-running and right-running rarefaction waves, respectively. Now, the local velocity increment $\delta u$ across a centered rarefaction wave becomes

$$
\delta u= \pm \int_{P_{0}}^{P} \frac{\mathrm{d} P}{\rho a}
$$

where the speed of sound for equilibrium gases can be calculated from

$$
a^{2}=\frac{\mathrm{d} P}{\mathrm{~d} \rho} \text {. }
$$

With $P$ known, the local flow velocity through a centered rarefaction wave becomes

(a)

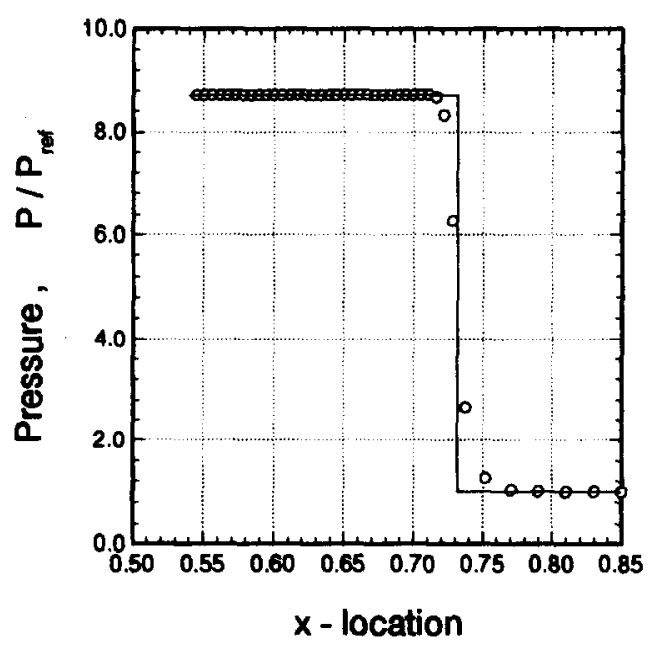

(c)

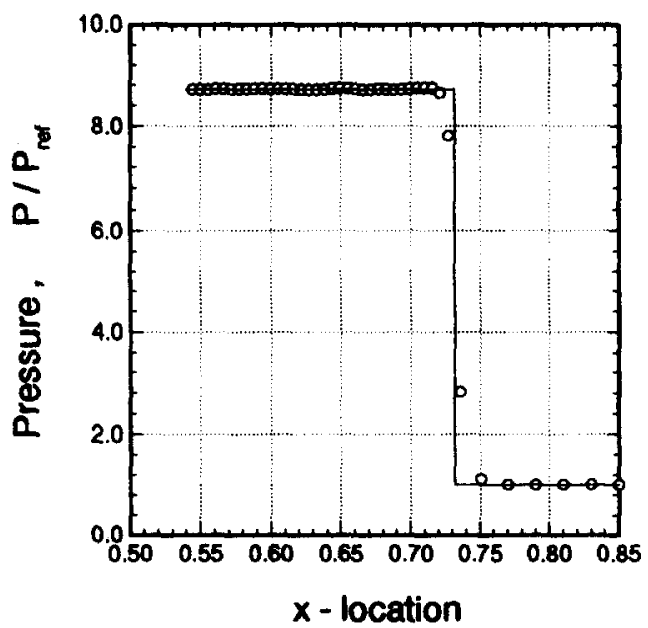

(b)

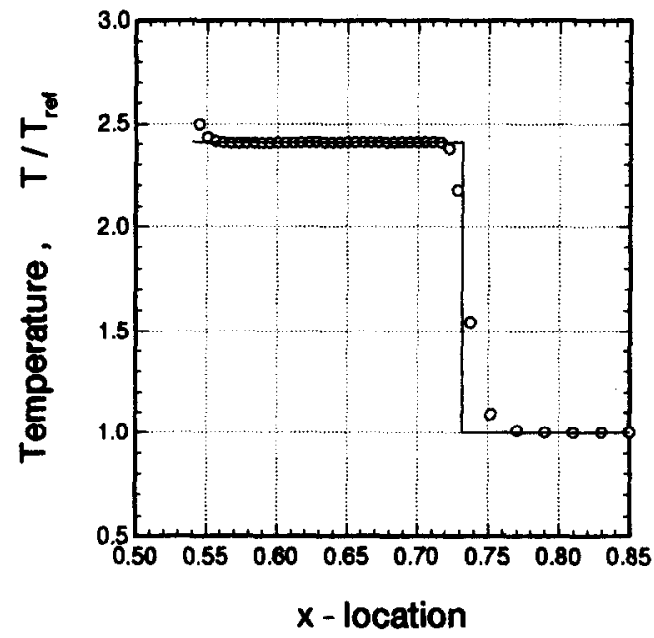

(d)

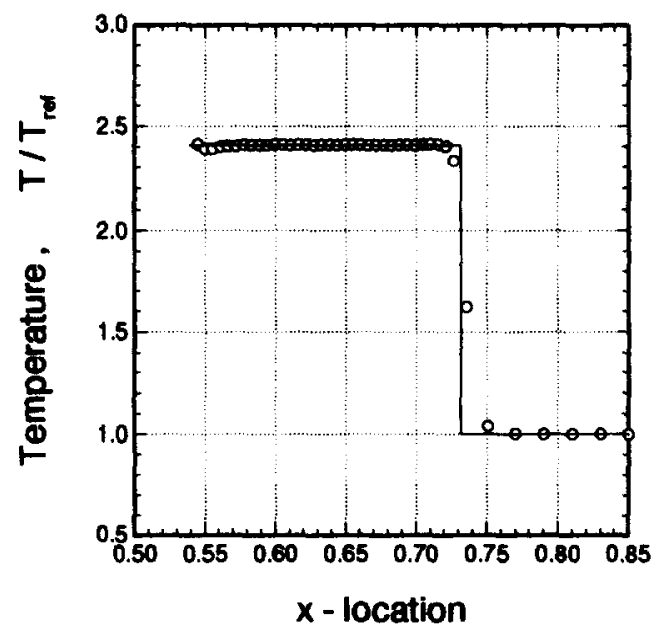

Fig. 6. Solution of forward piston problem with $U_{p}=2 U_{\text {ref }}$ for a perfect air at time $t=0.8 \mathrm{~ms}$. A comparison of numerical results (circles) and exact solution (solid-line). (a) and (b) First-order Godunov scheme; (c) and (d) second-order ENO scheme. 


$$
\left\{\begin{array}{l}
u=\Phi_{\mathrm{R}}(P)=u_{0}+\int_{P_{0}}^{P} \frac{\mathrm{d} P}{\rho a} \quad \text { (for the flow on the right) } \\
u=\Phi_{\mathrm{L}}(P)=u_{0}-\int_{P_{0}}^{P} \frac{\mathrm{d} P}{\rho a} \quad \text { (for the flow on the left) }
\end{array}\right.
$$

The integrals in Eq. (42) can be evaluated numerically using Simpson's rule.

Therefore, in the $\mathscr{L}^{E}(\Delta t)$ integration step, through any state $Q_{0}$, with $P / P_{0}$ as parameter, there are two families of states connecting to $Q_{0}$, namely, the compression state $\left(P / P_{0} \geqslant 1\right)$, and the expansion state $\left(P / P_{0} \leqslant 1\right)$. The two families join smoothly and can be regarded as a single family. For example, in the plane, the two curves form a single smooth curve at $Q=Q_{0}$, and in the $P-u$ plane, a constant $P=P^{*}$ line can be drawn and $u^{*}$ can be determined.

(a)

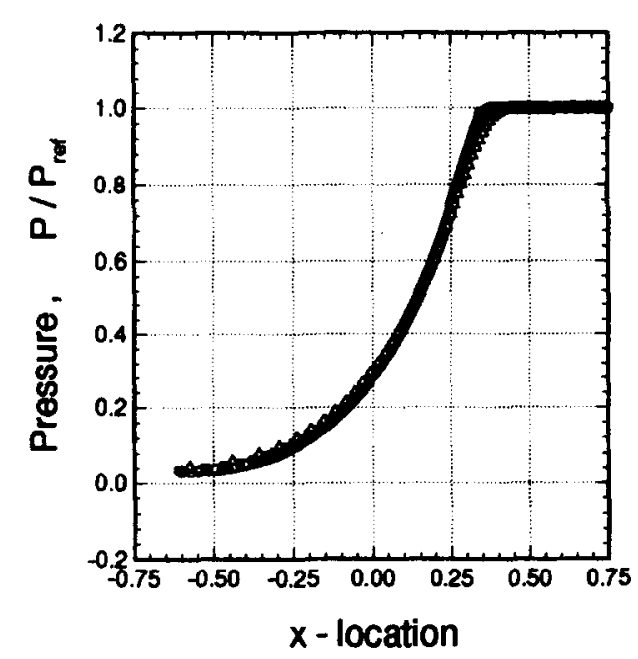

(c)

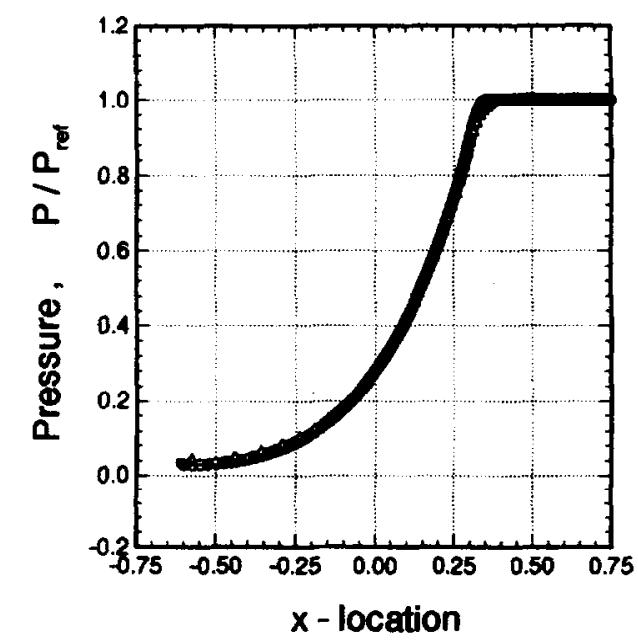

(b)

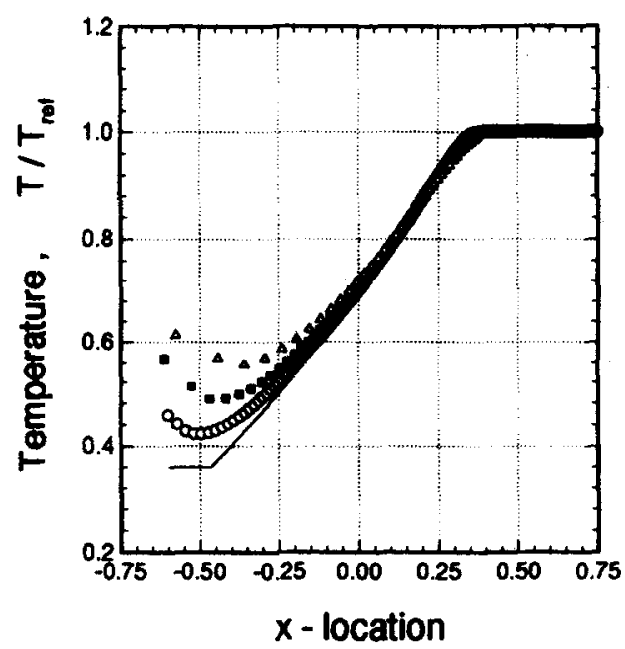

(d)

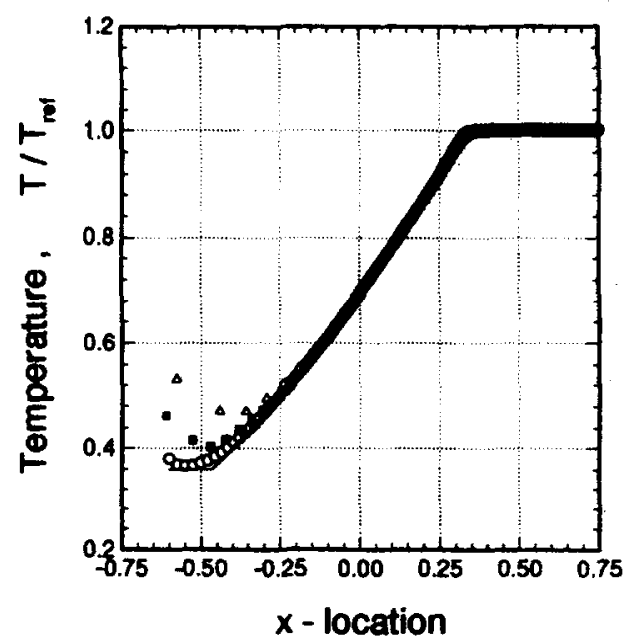

Fig. 7. Solution of backward piston problem with $U_{p}=-2 U_{\text {ref }}$ for a perfect air at time $t=0.6 \mathrm{~ms}$. A comparison of numerical results for $\Delta x_{\mathrm{ini}}=0.01$ (triangles), 0.005 (squares), 0.002 (circles), and exact solution (solid-line). (a) and (b) First-order Godunov scheme; (c) and (d) second-order ENO scheme. 


\subsection{Solution procedure of Riemann problem}

(i) In the $P-u$ plane, there are two curves that pass through the states $Q_{\mathrm{R}}$ and $Q_{\mathrm{L}}$; they are defined, respectively, by

$$
u=\Phi_{\mathrm{R}}(P)= \begin{cases}u_{\mathrm{R}}+\left(1-\frac{\rho_{\mathrm{R}}}{\rho}\right) \sqrt{\frac{P-P_{\mathrm{R}}}{\rho-\rho_{\mathrm{R}}} \frac{\rho}{\rho_{\mathrm{R}}}}, & P>P_{\mathrm{R}} \\ u_{\mathrm{R}}+\int_{P_{\mathrm{R}}}^{P} \frac{\mathrm{d} P}{\rho a}, & P \leqslant P_{\mathrm{R}}\end{cases}
$$

and

(a)

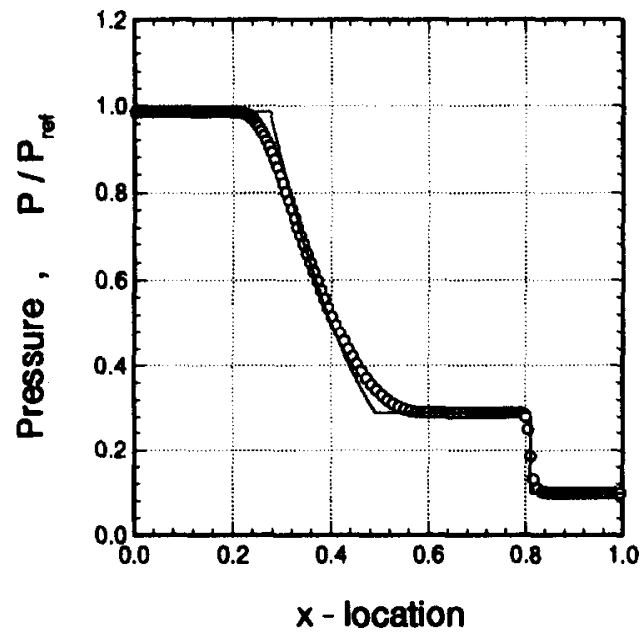

(c)

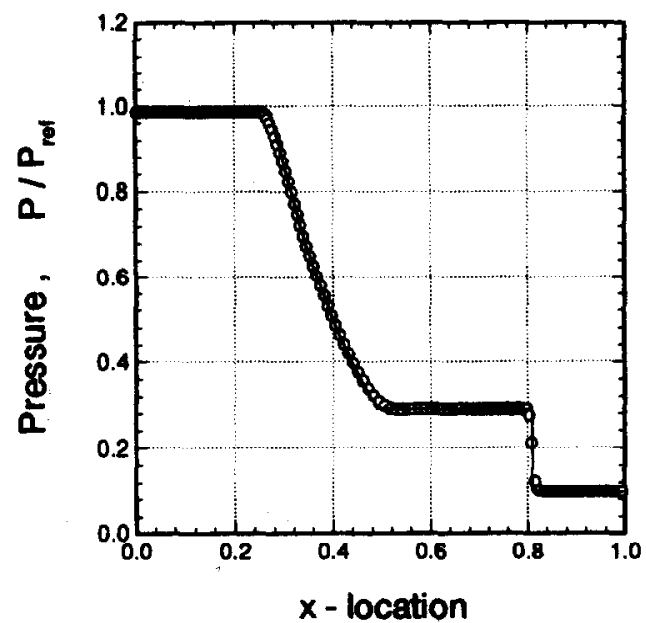

(b)

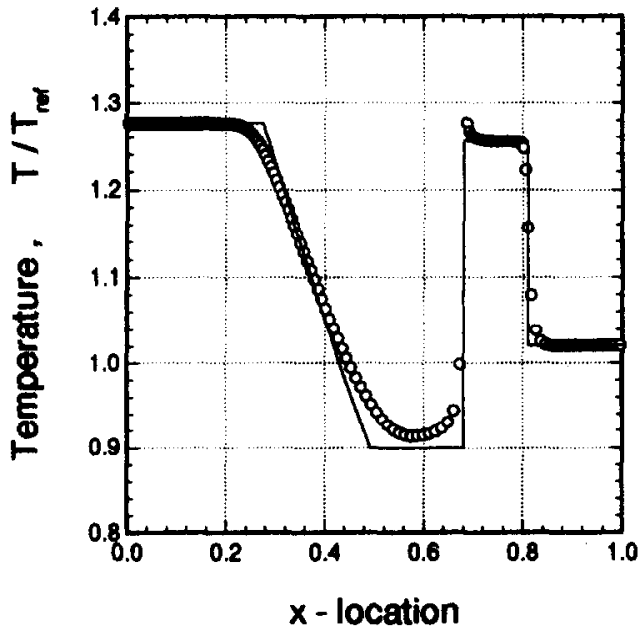

(d)

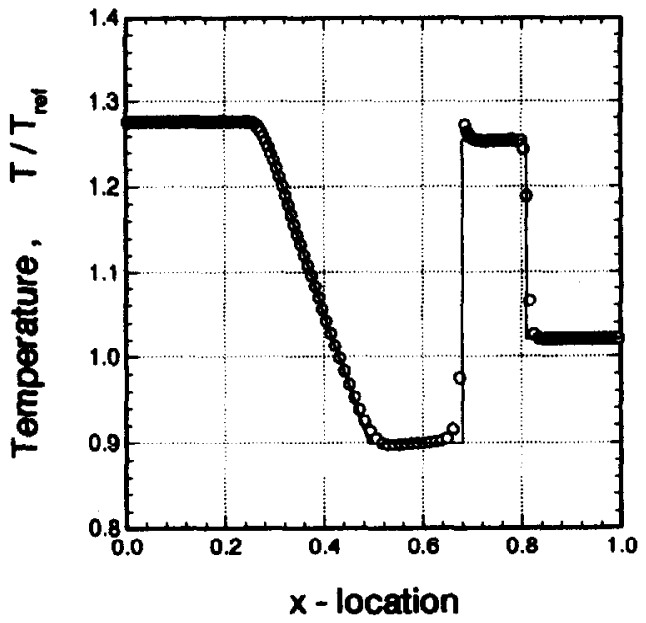

Fig. 8. Solution of Sod's first shock tube problem with two different ideal gases at time $t=0.6 \mathrm{~ms}$ using the ENO2 scheme. A comparison of numerical results (circles) and exact solution (solid-line). (a) Pressure; (b) temperature; (c) density; (d) velocity. 
$u=\Phi_{\mathrm{L}}(P)= \begin{cases}u_{\mathrm{L}}-\left(1-\frac{\rho_{\mathrm{L}}}{\rho}\right) \sqrt{\frac{P-P_{\mathrm{L}}}{\rho-\rho_{\mathrm{L}}} \frac{\rho}{\rho_{\mathrm{L}}}}, & P>P_{\mathrm{L}} \\ u_{\mathrm{L}}-\int_{P_{\mathrm{L}}}^{P} \frac{\mathrm{d} P}{\rho a}, & P \leqslant P_{\mathrm{L}}\end{cases}$

(ii) Using appropriate numerical procedure to solve $P^{*}$ and $u^{*}$. Define the object function as follows:

$$
f(P)=\Phi_{\mathrm{R}}(P)-\Phi_{\mathrm{L}}(P) .
$$

Then, the Riemann solution becomes

$$
f\left(P^{*}\right)=0 ; \quad u^{*}=\Phi_{\mathrm{R}}\left(P^{*}\right)=\Phi_{\mathrm{L}}(P) .
$$

(a)

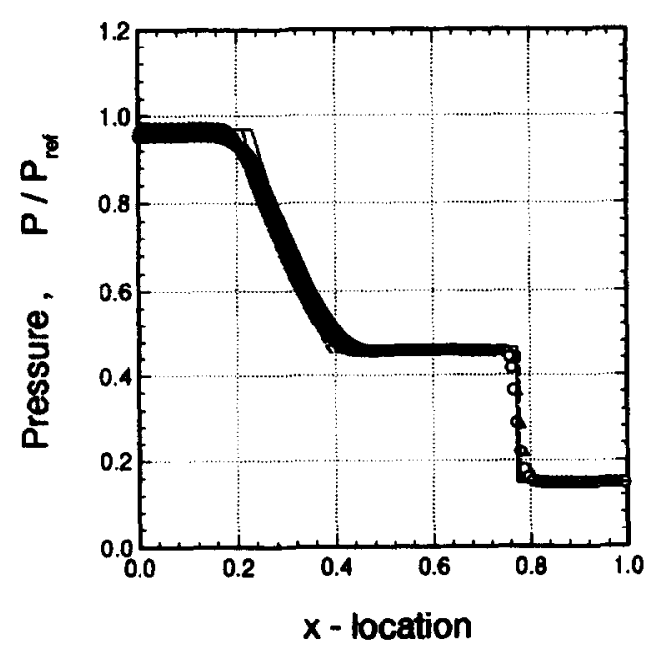

(c)

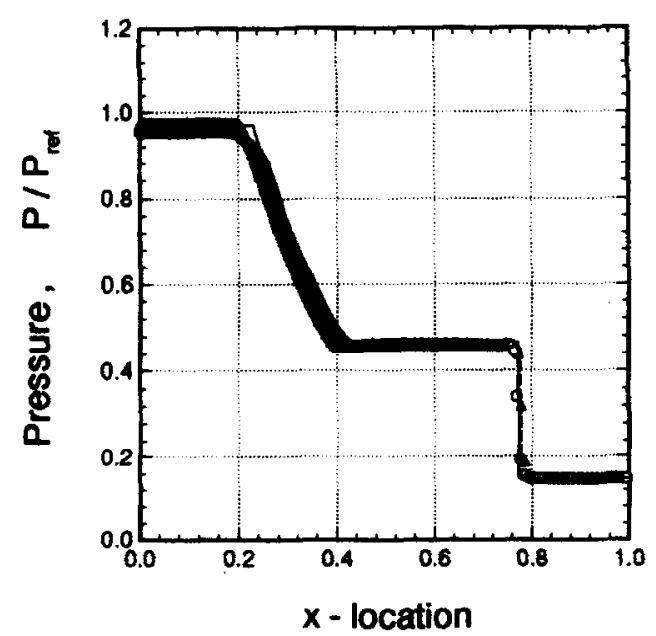

(b)

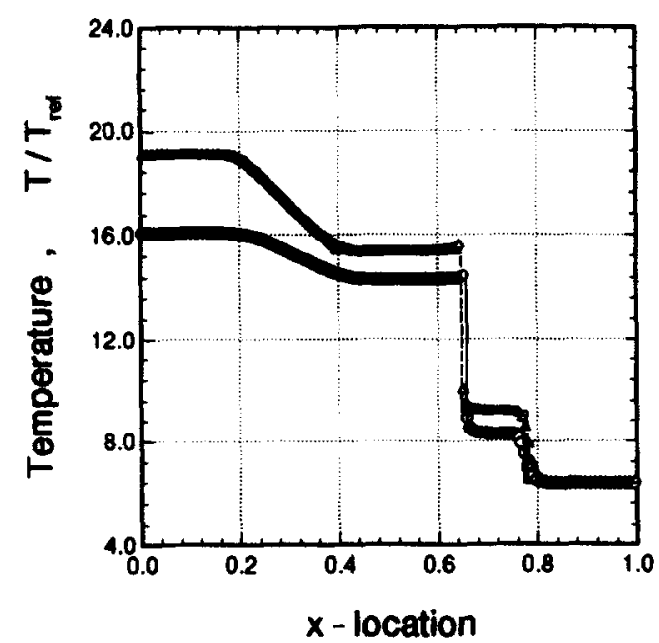

(d)

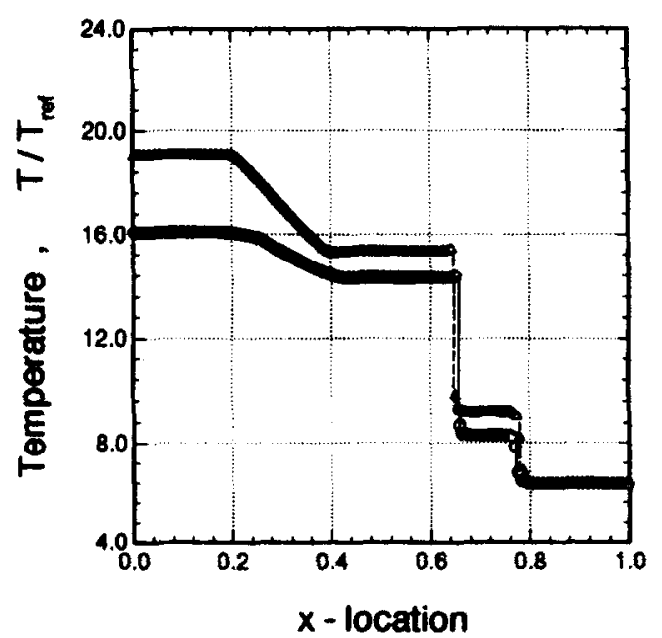

Fig. 9. Pressure and temperature results of a high-temperature shock tube flow for test case $A$ in [7] at time $t=0.2 \mathrm{~ms}$. A comparison of numerical results (circles) and exact solution (solid-line). (a) and (b) First-order Godunov scheme; (c) and (d) second-order ENO scheme. 
(iii) With the values $P^{*}$ and $u^{*}$ known, we can calculate the numerical fluxes $G_{i \pm 1 / 2}^{N}$ such that

$$
G_{i \pm 1 / 2}^{N}=\left(\begin{array}{c}
0 \\
u^{*} \\
u^{*} P^{*}
\end{array}\right),
$$

in our pathline Godunov method, from the definition of the numerical fluxes $\boldsymbol{G}_{i \pm 1 / 2}^{N}$ and $s_{i \pm 1 / 2}^{0}=\left(u^{*}\right)_{i \pm 1 / 2}^{n+1 / 2}$. Eq. (47) implies that we do not need to compute the other flow variables on both sides of the contact discontinuity.

At a solid endwall the condition of flow velocity matching requires that the solid endwall is also a pathline. This causes a boundary Riemann problem and is specified as the system (1) of hyperbolic conservation laws subject to the condition

(a)

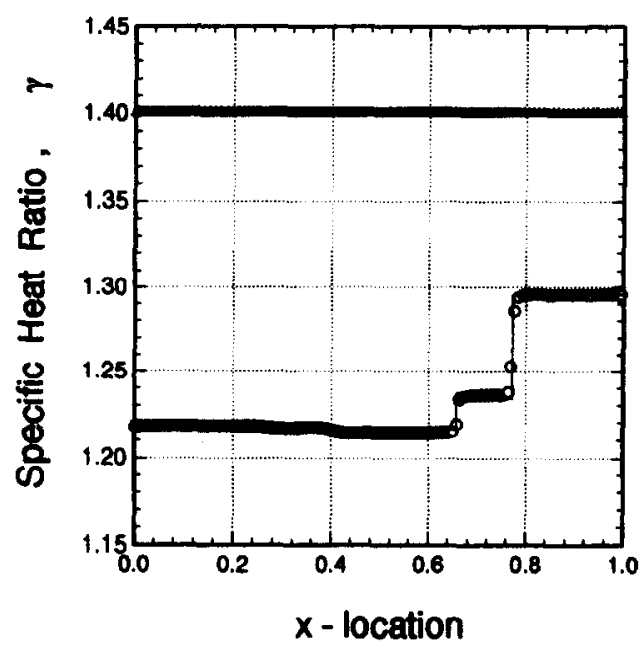

(c)

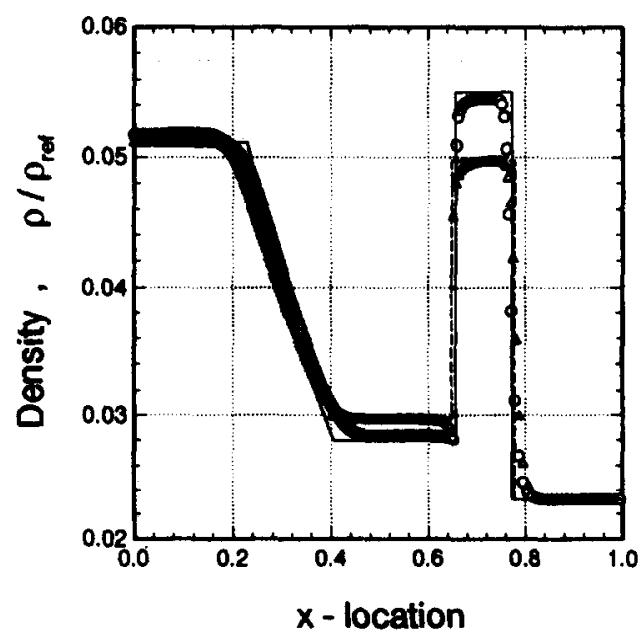

(b)

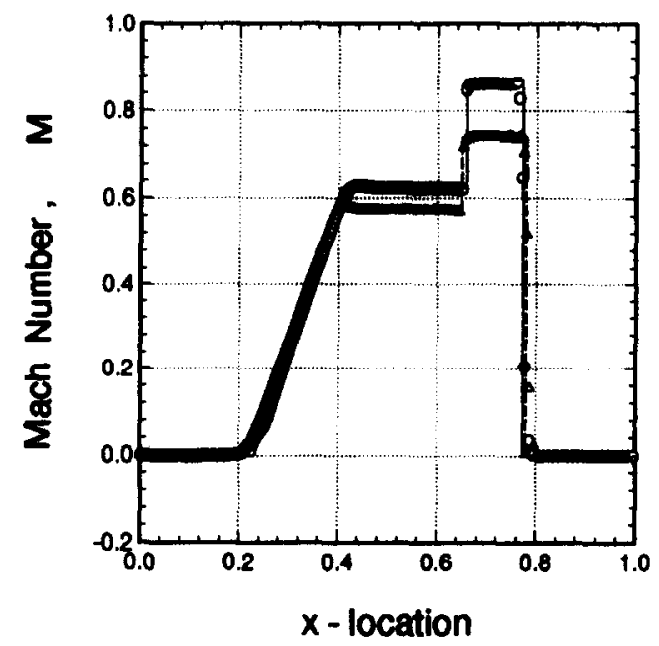

(d)

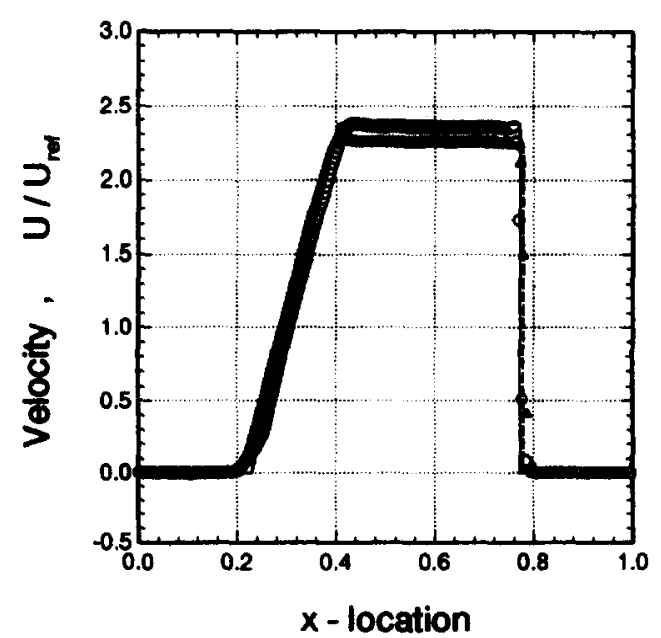

Fig. 10. Other thermodynamic properties of a high-temperature shock tube flow for test case A in [7] using the ENO2 scheme at time $t=0.2 \mathrm{~ms}$. A comparison of numerical results for perfect air (triangles) and equilibrium air (circles) and exact solution for perfect air (dash-line) and equilibrium air (solid-line). (a) Specific heat ratio; (b) Mach number; (c) density; (d) velocity. 


$$
\begin{cases}Q\left(x, t=t^{n}\right)=Q_{\mathrm{R}}, & x>x_{w} \\ u=u^{*}=u_{w}, & x=x_{w}\end{cases}
$$

Here, we assume that the flow is on the right of solid endwall. In this way the condition of flow velocity matching is automatically satisfied. The solution procedure is then the same as that described above. That is, since

$$
u_{w}=\Phi_{\mathrm{R}}(P)= \begin{cases}u_{\mathrm{R}}+\left(1-\frac{\rho_{\mathrm{R}}}{\rho}\right) \sqrt{\frac{P-P_{\mathrm{R}}}{\rho-\rho_{\mathrm{R}}} \frac{\rho}{\rho_{\mathrm{R}}}}, & u_{w}>u_{\mathrm{R}} \\ u_{\mathrm{R}}+\int_{P_{\mathrm{R}}}^{P} \frac{\mathrm{d} P}{\rho a}, & u_{w} \leqslant u_{\mathrm{R}}\end{cases}
$$

then, the boundary Riemann solution becomes

$$
f_{w}\left(P^{*}\right)=\Phi_{\mathrm{R}}\left(P^{*}\right)-u_{w}=0 ; \quad u^{*}=u_{w},
$$

for the flow on the right of solid endwall. A similar result can be obtained for the flow on the left of solid endwall.

The above elementary solutions of the unsteady Riemann problem for equilibrium gases are used in our pathline Godunov method described in the previous section. And the Riemann solver discussed here can be also used to obtain the exact solution for some of the basic test problems for the perfect and equilibrium flows.

(a) Perfect Air

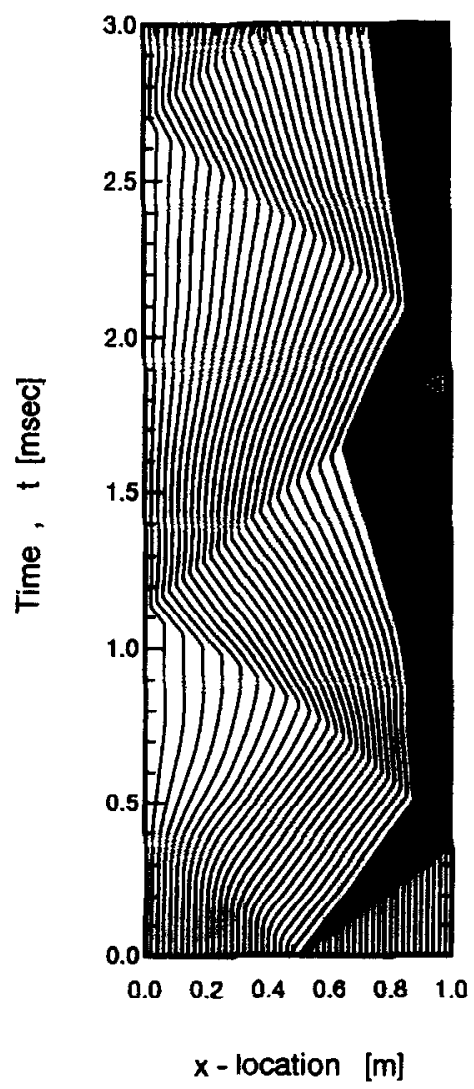

(b) Equilibrium Air

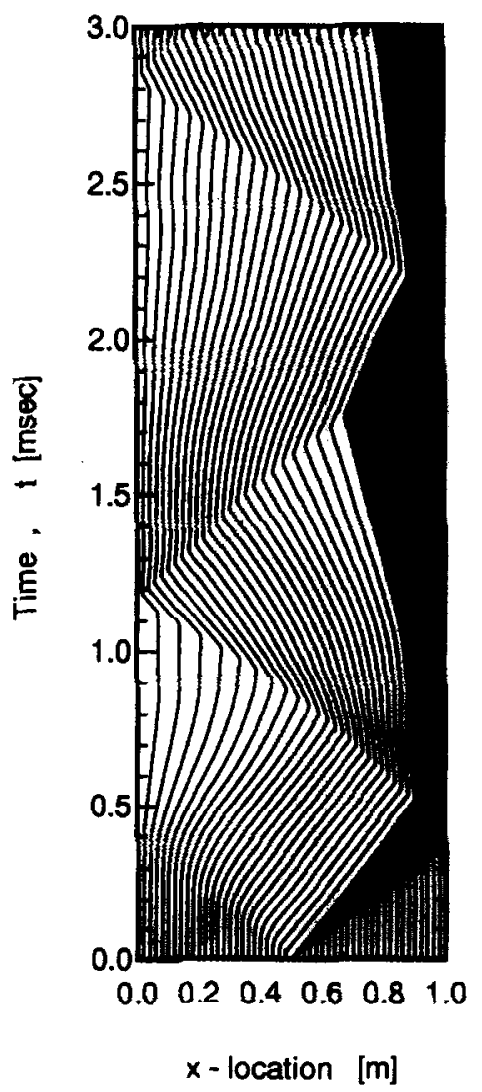

Fig. 11. Pathline grids of a high-temperature shock tube flow for test case A in [7] using the ENO2 scheme. (a) Perfect air flow; (b) equilibrium air flow. 


\section{Numerical results and discussions}

In this section, we test the performance of the present second-order essentially nonoscillatory pathline Godunov method by solving several simple initial and boundary value Riemann problems of unsteady equilibrium flows and comparing the results with exact solutions. Computations of practical unsteady quasi-onedimensional equilibrium flows are also included. All computations were done with a uniform grid at the initial marching step and with $\mathrm{CFL}=1.0$. Also note that the reference conditions used in all computational cases are: $P_{\text {ref }}=101330 \mathrm{~N} / \mathrm{m}^{2}, \rho_{\text {ref }}=1.292 \mathrm{Kg} / \mathrm{m}^{3}, U_{\text {ref }}=331 \mathrm{~m} / \mathrm{s}, T_{\text {ref }}=273^{\circ} \mathrm{K}$, and then $h_{0, \text { ref }}$ and other variables are respectively calculated from these basic reference conditions.

The first example is a model test case of initial-value Riemann problem. It is formed by an ideal shock tube problem for air taken as a perfect gas with $\gamma=1.4$ and with diaphragm initially located at $x_{0}=0.5$. The initial condition (all data in SI units) is given by

$$
\begin{cases}Q_{\mathrm{L}}=\left(\rho_{\mathrm{L}}, u_{\mathrm{L}}, P_{\mathrm{L}}\right)=\left(1.000,0.0,10^{5}\right), & 0<x<0.5 \\ Q_{\mathrm{R}}=\left(\rho_{\mathrm{R}}, u_{\mathrm{R}}, P_{\mathrm{R}}\right)=\left(0.125,0.0,10^{4}\right), & 0.5<x<1.0\end{cases}
$$

This test data corresponds to those applied by Sod [17]. In the exact solution, the resulting interaction produces a moving normal shock wave, a centered rarefaction wave, and a contact discontinuity in between. Here, we use 100 uniform cells with initial cell size $\Delta x_{\mathrm{ini}}=0.01$. Numerical results for the flow properties at time $t=0.6 \mathrm{~ms}$

(a) Perfect Air

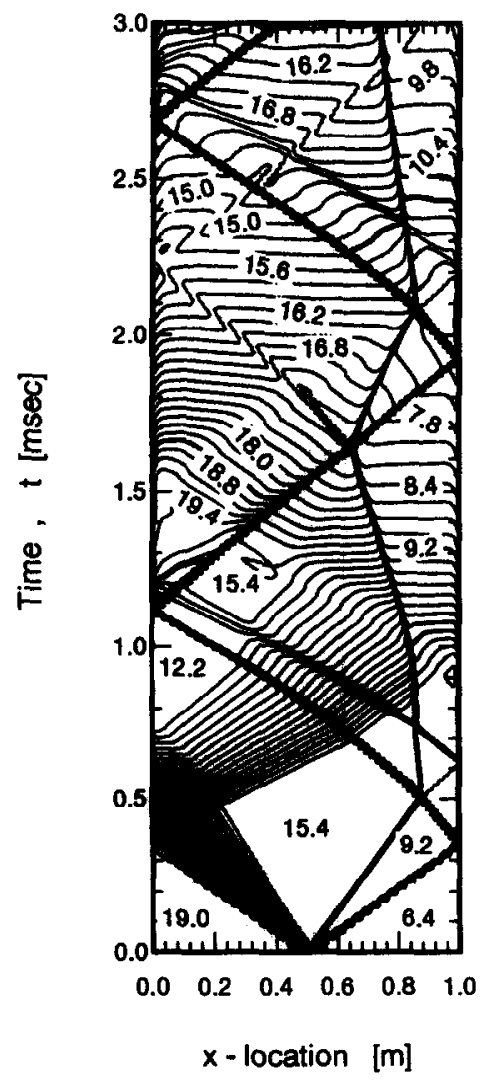

(b) Equilibrium Air

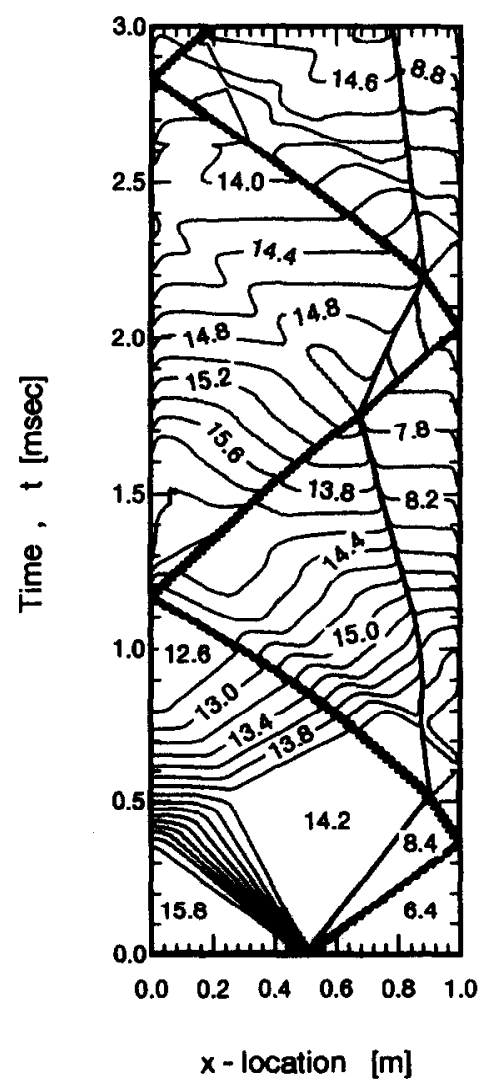

Fig. 12. Time history of temperature-contour distribution of a high-temperature shock tube flow for test case A in [7] using the ENO2 scheme. (a) Perfect air flow; (b) equilibrium air flow. 
are shown in Fig. 4 along with the exact solution which was obtained using the real gas Riemann solver described in Section 4. Results obtained using both first-order Godunov scheme and ENO2 scheme are shown. The ENO2 results indicate better resolution at shocks and rare faction wave structure. By using the pathline mesh system, a better resolution of contact discontinuity, even for the first-order scheme, can be obtained and is the important feature as compared with other Cartesian meshes.

Next, we consider another unsteady Riemann problem which is similar to the above test case but with greater pressure and density ratios. The initial condition (all data in SI units) is given by

$$
\begin{cases}Q_{\mathrm{L}}=\left(\rho_{\mathrm{L}}, u_{\mathrm{L}}, P_{\mathrm{L}}\right)=\left(1.00,0.0,10^{5}\right), & 0<x<0.5 \\ \boldsymbol{Q}_{\mathrm{R}}=\left(\rho_{\mathrm{R}}, u_{\mathrm{R}}, P_{\mathrm{R}}\right)=\left(0.01,0.0,10^{3}\right), & 0.5<x<1.0\end{cases}
$$

(a)

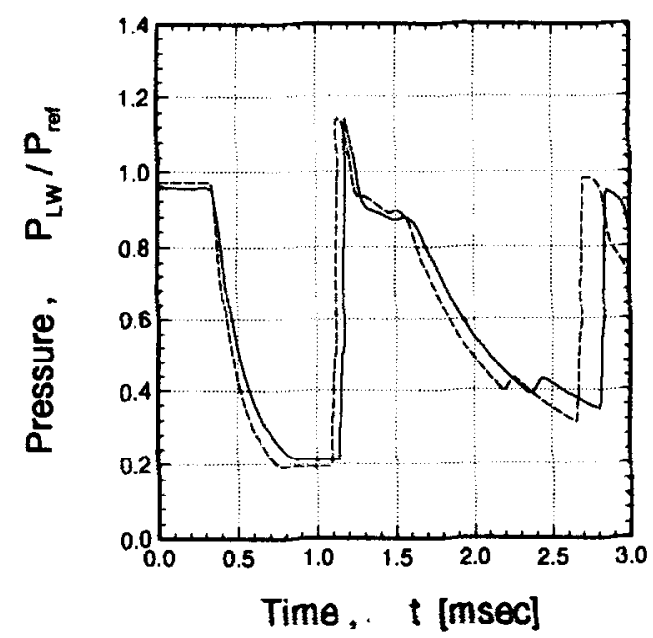

(c)

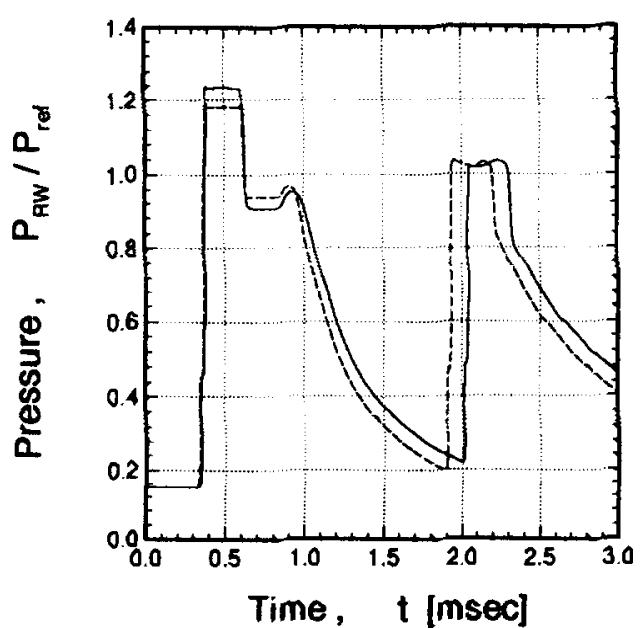

(b)

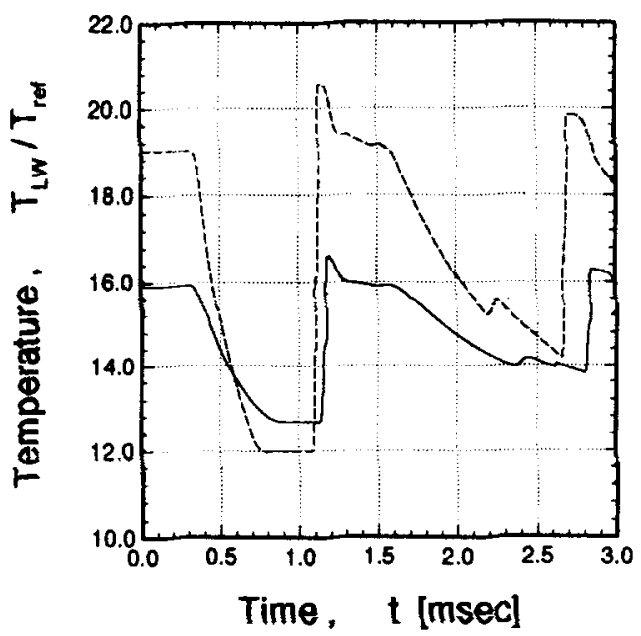

(d)

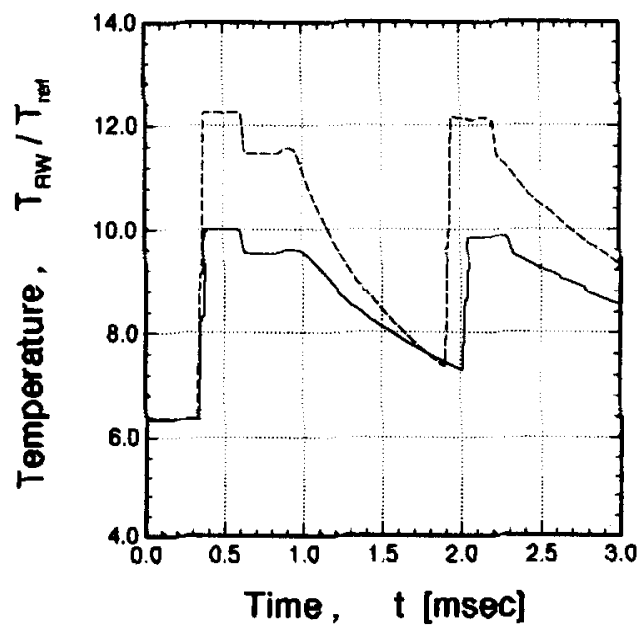

Fig. 13. Time history of surface flow properties at the left and right solid endwalls for test case A in [7] using the ENO2 scheme. A comparison of perfect flow (dash-line) and equilibrium flow (solid-line). (a) and (b) At the left solid endwall; (c) and (d) at the right solid endwall. 
This is a more severe case than the above first basic test case of Riemann problem, but the resulting interaction produces similar features, namely a moving normal shock adjacent to the low-pressure stream side and a centered rarefaction flow on the high-pressure stream side. In this case we use 250 cells at high pressure side and 50 cells at low pressure side with the different initial cell size $\Delta x_{\text {ini }}=0.002$ and 0.01 , respectively. Numerical results for the flow properties at time $t=0.4 \mathrm{~ms}$ are shown in Fig. 5 along with the exact solution which was generated also using the real gas Riemann solver. Again, it is seen that shock and contact discontinuities are still sharply resolved by our Godunov-type scheme based on the pathline meshes. In the same manner, the ENO2 results give a better resolution at shock, contact discontinuity, and also rarefaction wave structure. It is also noted, that the application of ENO2 scheme can improve greatly the accuracy of thermodynamic properties (such as $\mathrm{T}$ ) within the uniform region behind the normal shock wave.

As an example of a simple initial and boundary value Riemann problem we compute the flow generated by a piston moving impulsively into a quiescent gas with a constant speed. That is, a piston with $U_{p}=2 U_{\text {ref }}$ moves

(a)

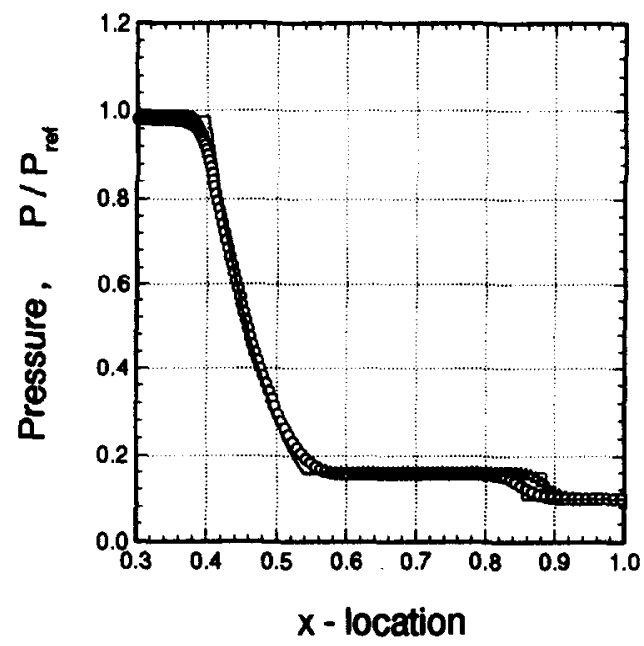

(c)

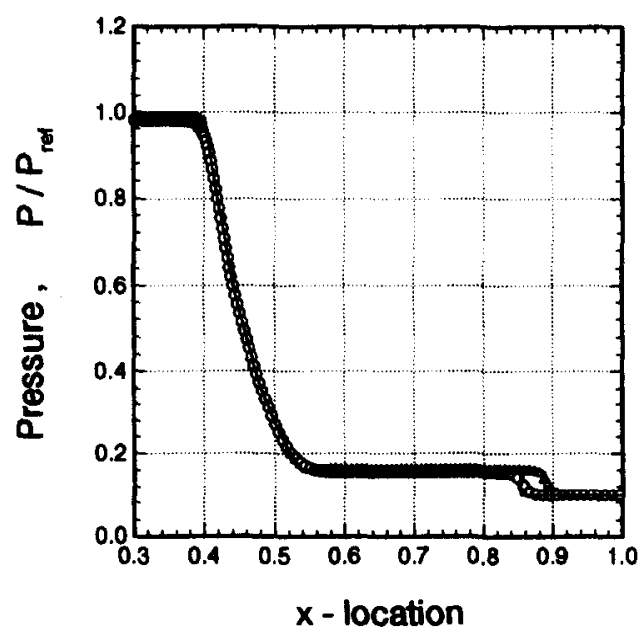

(b)

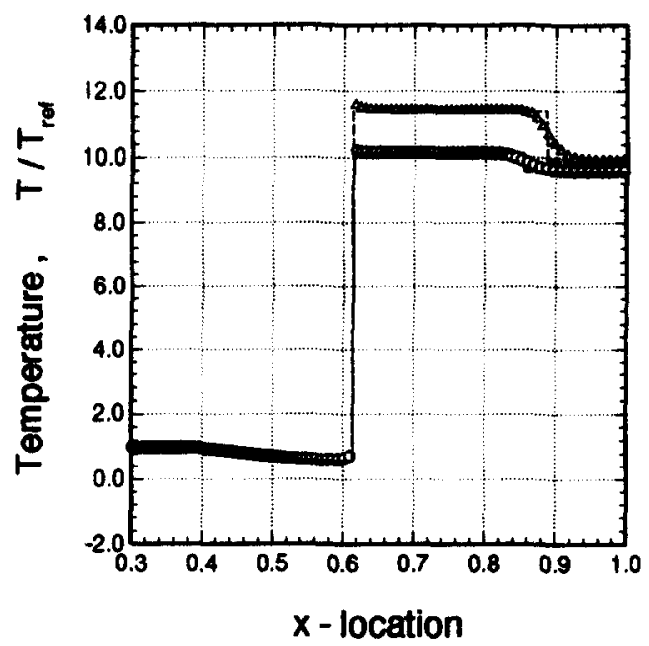

(d)

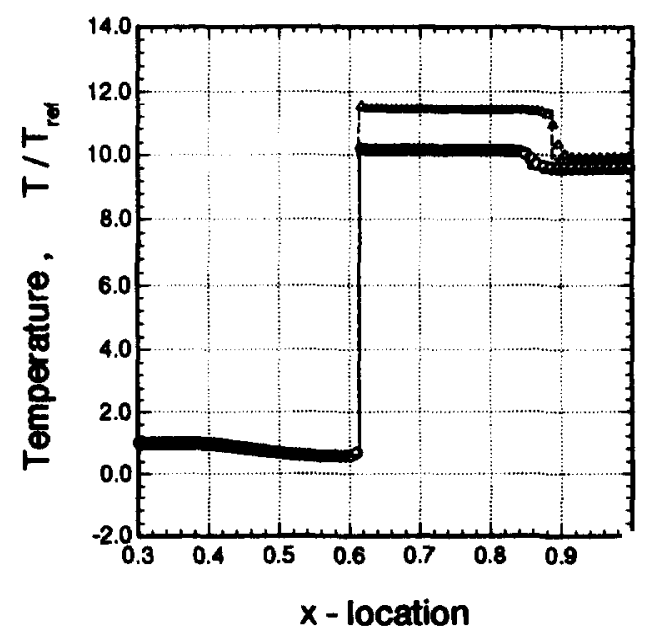

Fig. 14. Pressure and temperature results of a high-temperature shock tube flow for test case $\mathrm{C}$ in [17] at time $t=0.3 \mathrm{~ms}$. A comparison of numerical results for perfect air (triangles) and equilibrium air (circles) and exact solution for perfect air (dash-line) and equilibrium air (solid-line). (a) and (b) First-order Godunov scheme; (c) and (d) second-order ENO scheme. 
forward into a perfect air with one atmosphere filled within a constant-area circular tube. The gas within tube suffers a compression process. In this case, 50 cells and $\Delta x_{\text {ini }}=0.02$ was used. Numerical results for the flow properties at time $t=0.8 \mathrm{~ms}$ are plotted in Fig. 6 and compared with the exact solution which was obtained using the boundary Riemann solver described in Section 4. Good accuracy for the flow properties within the uniform region behind the moving normal shock and a better resolution at shock are illustrated.

Another simple initial-boundary-value Riemann problem we computed was the flow generated by an impulsively withdrawing piston from the gas with a constant speed. That is, a piston with $U_{p}=-2 U_{\text {ref }}$ withdrawn from a perfect air with one atmosphere filled within a constant-area circular tube. The gas within the tube suffers an expansion process. Here 100,200 and 500 cells are employed with $\Delta x_{\text {ini }}=0.01,0.005,0.002$, respectively. Numerical results for the flow properties at time $t=1.0 \mathrm{~ms}$ are plotted in Fig. 7 and compared with the exact solution which was also obtained using the boundary Riemann solver. Due to the intrinsic feature of

(a)

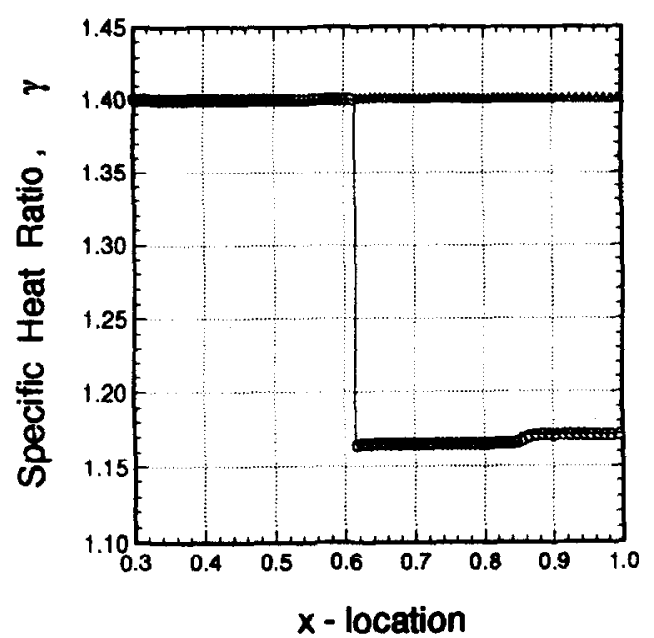

(c)

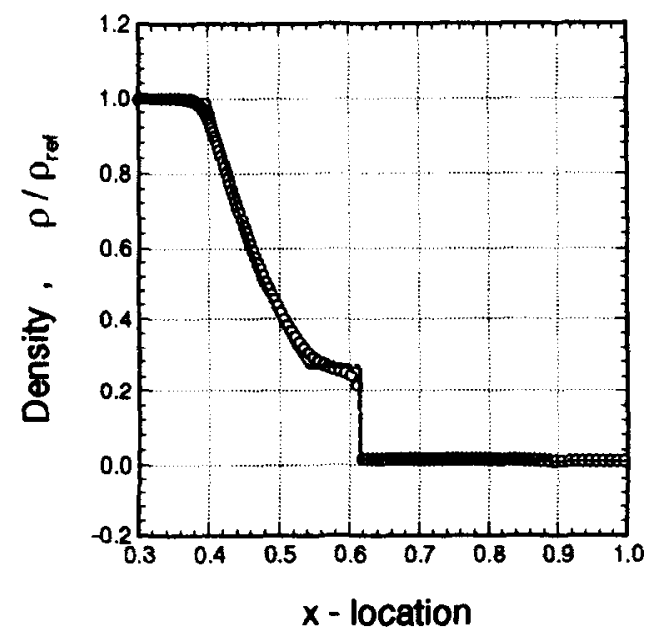

(b)

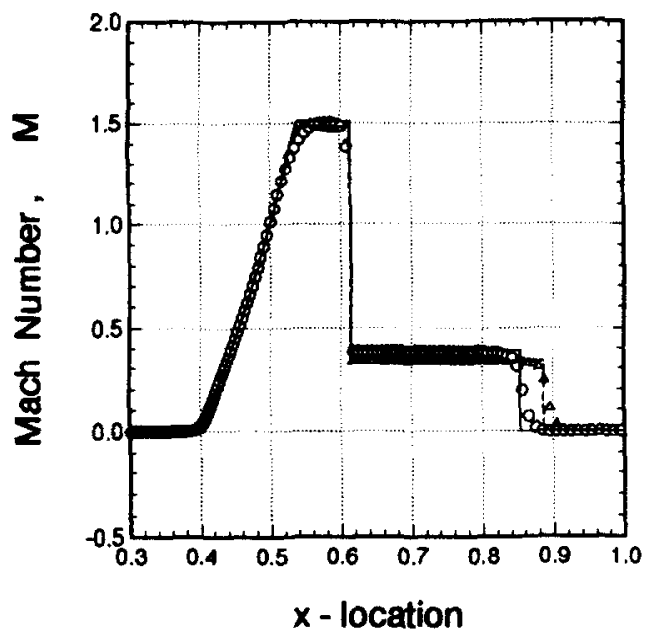

(d)

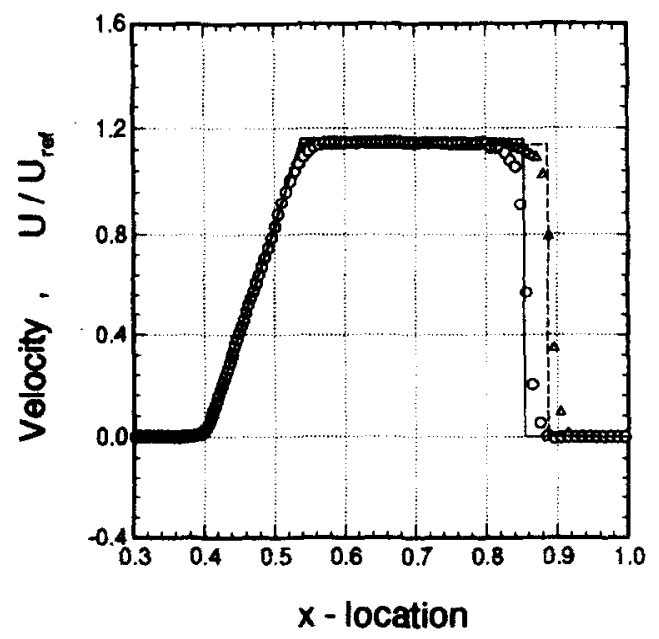

Fig. 15. Other thermodynamic properties of a high-temperature shock tube flow for test case C in [7] using the ENO2 scheme at time $t=0.3 \mathrm{~ms}$. A comparison of numerical results for perfect air (triangles) and equilibrium air (circles) and exact solution for perfect air (dash-line) and equilibrium air (solid-line). (a) Specific heat ratio; (b) Mach number; (c) density; (d) velocity. 
pathline, grid size should be wider after rarefaction and the accuracy of computation could deteriorate, especially at the tail of rarefaction wave. To attain the same level of accuracy in representation of rarefaction wave structure, the use of refined grids to recover the same grid size after flow rarefaction is a good way of remedy, as can be seen from Fig. 7. Otherwise, with the application of the ENO2 scheme, a great improvement in computational accuracy can be achieved, as is evident in Fig. 7.

To apply the pathline Godunov method to multi-component shock tube flows, we next consider the same first Sod's shock tube test problem with two different ideal gases. The initial data given to this unsteady Riemann problem, relative to basic reference conditions, is

$$
\left\{\begin{array}{lll}
Q_{\mathrm{L}}=\left(\rho_{\mathrm{L}}, u_{\mathrm{L}}, P_{\mathrm{L}}\right)=(1.000,0.0,1.0) ; & \gamma_{\mathrm{L}}=1.4, & 0<x<0.5 \\
\boldsymbol{Q}_{\mathrm{R}}=\left(\rho_{\mathrm{R}}, u_{\mathrm{R}}, P_{\mathrm{R}}\right)=(0.125,0.0,0.1) ; & \gamma_{\mathrm{R}}=1.2, & 0.5<x<1.0
\end{array}\right.
$$

The same problem has been considered by Karni [18]. All the computational conditions are the same as the previous example for the first Sod's shock tube problem. Numerical results for the flow properties at time $t=0.6 \mathrm{~ms}$ are shown in Fig. 8 along with the exact solution which was obtained using the real gas Riemann solver, described in Section 4 also. For this multi-component fluid flow, it was reported that due to the erroneous pressure fluctuations generated by the conservative wave model, strong oscillations and other computational inaccuracy near material interfaces in the solution of the extended Euler models in conservation form may appear [18]. In Fig. 8, numerical results show good accuracy for the continuity of the pressure and particle velocity in contact discontinuity. This indicates that, at least for this problem, our pathline meshes can easily

(a) Perfect Air

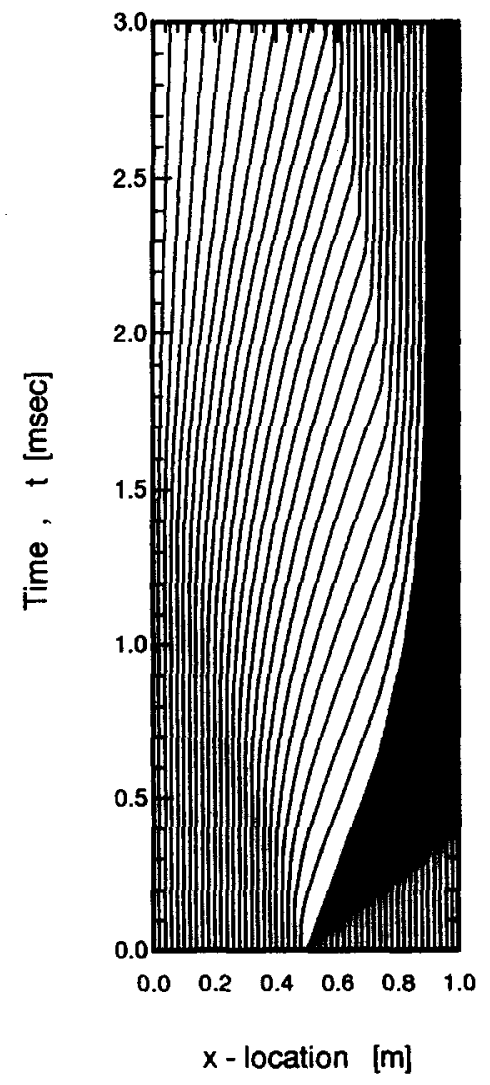

(b) Equilibrium Air

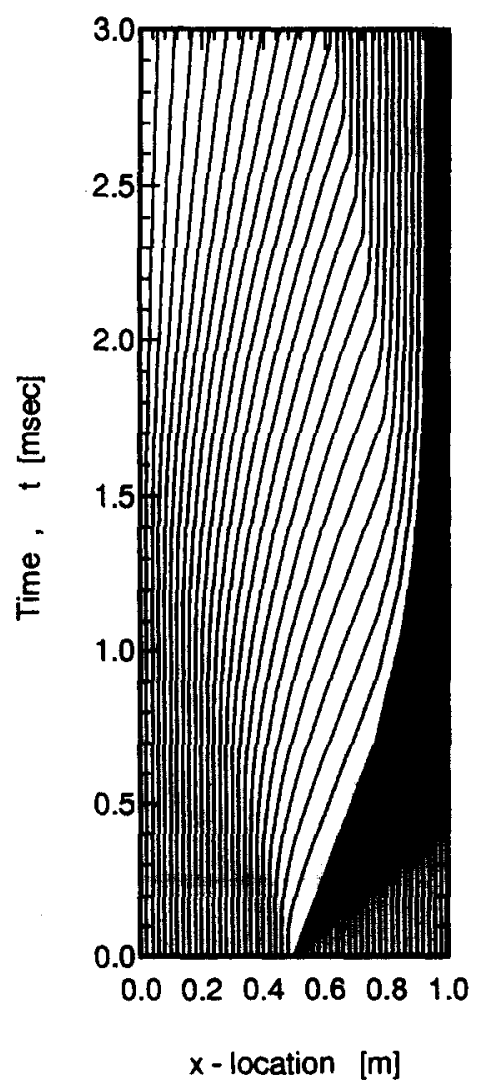

Fig. 16. Pathline grids of a high-temperature shock tube flow for test case $\mathrm{C}$ in [7] using the ENO2 scheme. (a) Perfect air flow; (b) equilibrium air flow. 
obtain a good resolution for the flow properties in the whole flowfield with multi-component shock tube flows.

Another example considered is that of high-temperature shock tube flow with initial condition (all data in SI units) given by

$$
\begin{cases}Q_{\mathrm{L}}=\left(\rho_{\mathrm{L}}, u_{\mathrm{L}}, P_{\mathrm{L}}\right)=\left(0.0660,0.0,9.84 \times 10^{4}\right), & 0<x<0.5 \\ Q_{\mathrm{R}}=\left(\rho_{\mathrm{R}}, u_{\mathrm{R}}, P_{\mathrm{R}}\right)=\left(0.0300,0.0,1.50 \times 10^{4}\right), & 0.5<x<1.0\end{cases}
$$

This is the test case A applied by Montagné et al. [7], which consists of both the real gas effect and multi-component gas problem. Although this case corresponds to significant variations of the thermodynamic properties of the gas, the condition is still not very severe. Here, we use 250 cells at high pressure side and 100 cells at low pressure side with different initial cell sizes $\Delta x_{\text {ini }}=0.002$ and 0.005 , respectively. The properties of pressure and temperature computed with both first-order Godunov and second-order ENO schemes along with the exact solution are shown in Fig. 9 at time $t=0.2 \mathrm{~ms}$. Obviously, the ENO2 results can achieve a better resolution at the shocks and for the rarefaction wave. Other flow properties computed with the second-order ENO scheme are also shown along with the exact solution in Fig. 10 at time $t=0.2 \mathrm{~ms}$. All numerical results agree well with the exact solutions. It can be clearly seen that the moving speed of normal shock and temperature behind the normal shock are smaller for the real gas, but the shock strength and the Mach number and flow velocity behind the moving normal shock are also higher. The computed pathline grids and the time history of temperature-contour distribution for the perfect and equilibrium air flows using ENO2 scheme are presented in Figs. 11 and 12. The gridlines clearly indicate also that the computational mesh follows exactly the

(a) Perfect Air

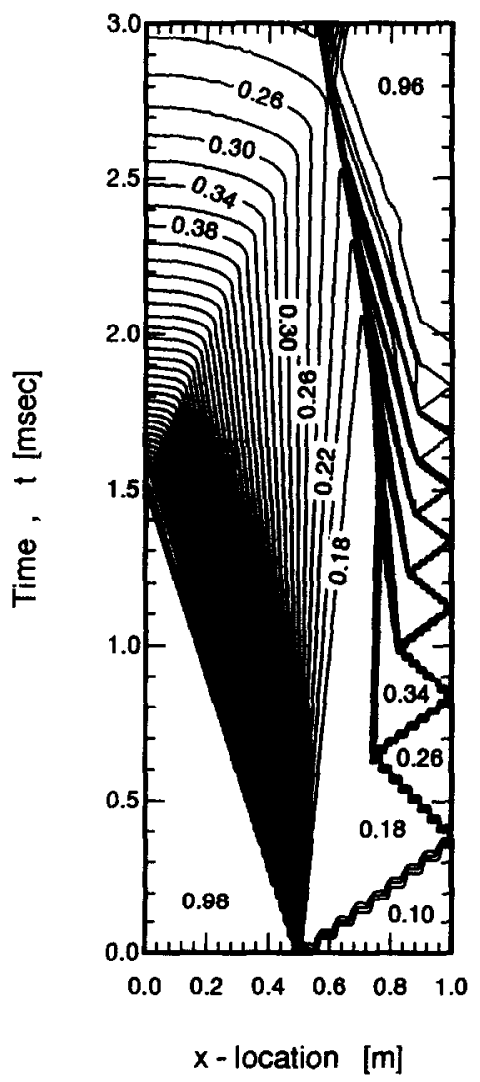

(b) Equilibrium Air

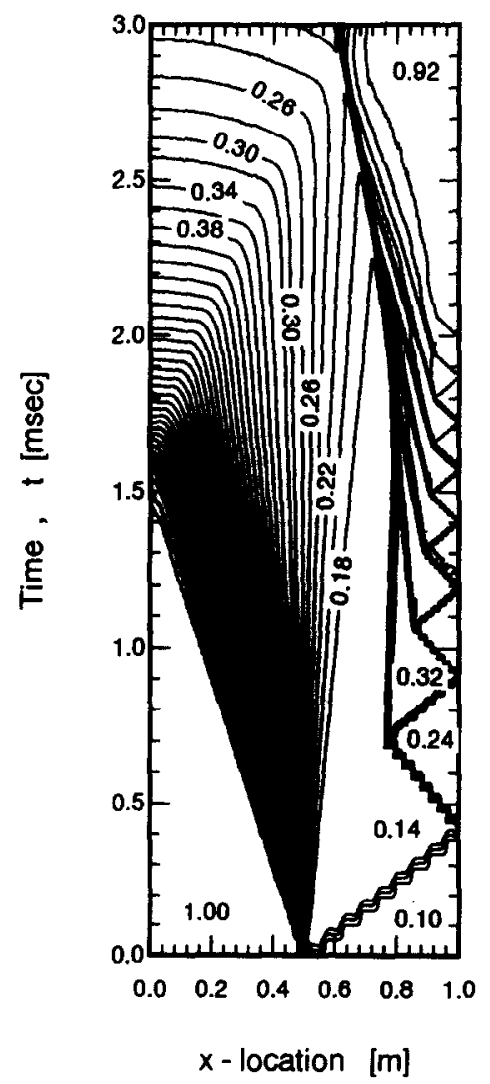

Fig. 17. Time history of pressure-contour distribution of a high-temperature shock tube flow for test case C in [7] using the ENO2 scheme. (a) Perfect air flow; (b) equilibrium air flow. 
particle movement even when it crosses a shock wave. The clustering of the grids at high gradient region such as shock wave can also be observed. The time history of the surface flow properties at the left and right solid endwalls for perfect and equilibrium air gases are also shown in Fig. 13.

Since real gas effects occur in general in flows with large discontinuities, it is important to identify this effect in order to estimate the performances of our pathline Godunov method for real gases. Next, we consider a high-temperature shock tube flow corresponds to a large jump of internal energy through the contact discontinuity. The initial condition (all data in SI units) given by

$$
\begin{cases}Q_{\mathrm{L}}=\left(\rho_{\mathrm{L}}, u_{\mathrm{L}}, P_{\mathrm{L}}\right)=\left(1.2900,0.0,1.0 \times 10^{5}\right), & 0<x<0.5 \\ Q_{\mathrm{R}}=\left(\rho_{\mathrm{R}}, u_{\mathrm{R}}, P_{\mathrm{R}}\right)=\left(0.0129,0.0,1.0 \times 10^{4}\right), & 0.5<x<1.0\end{cases}
$$

(a)

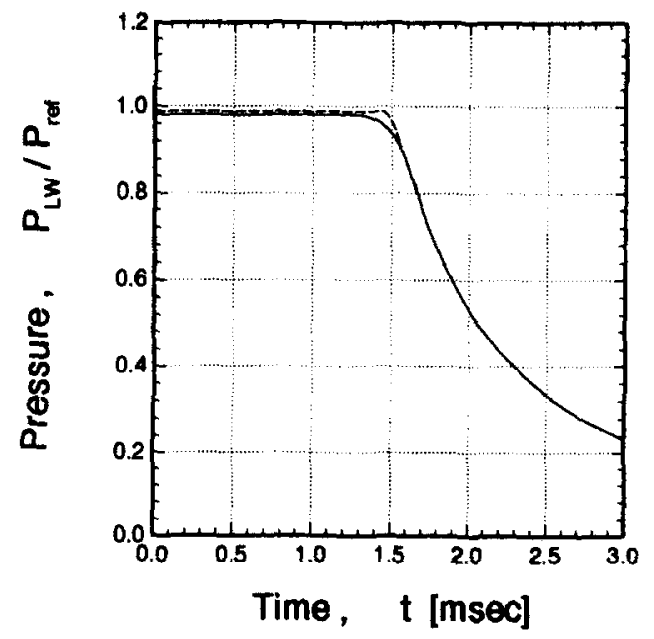

(c)

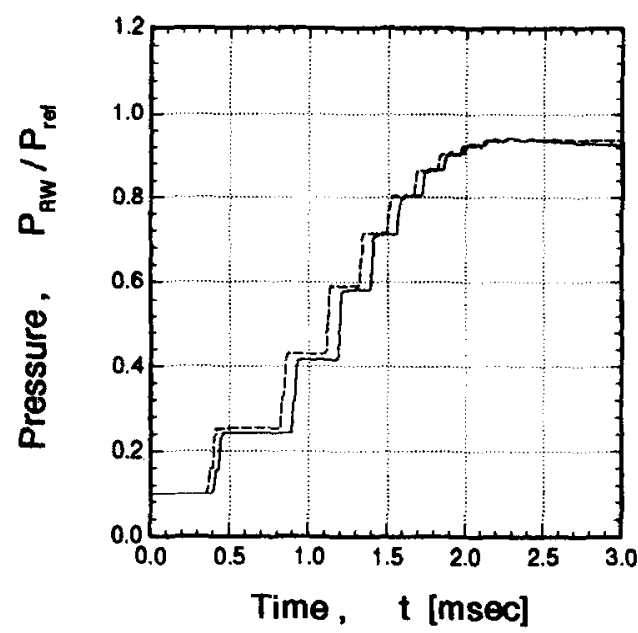

(b)

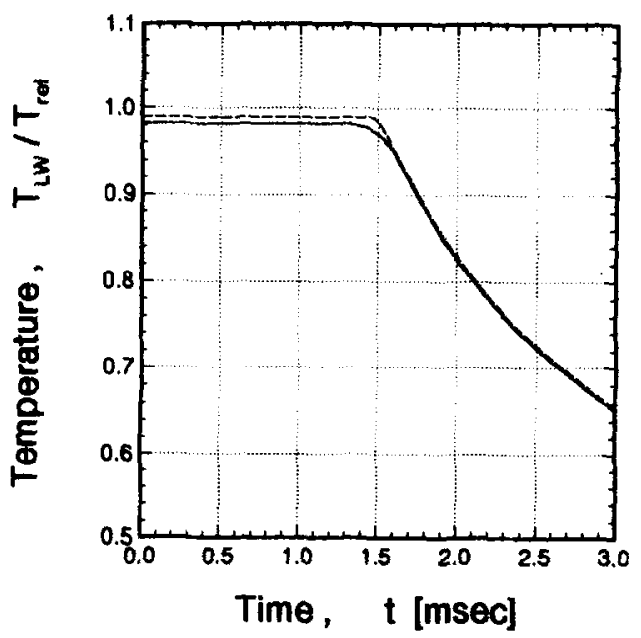

(d)

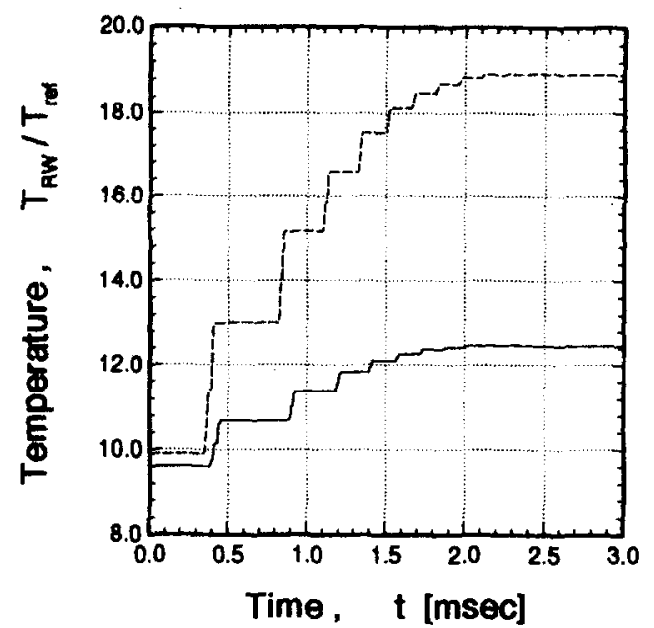

Fig. 18. Time history of surface flow properties at the left and right solid endwalls for test case $\mathrm{C}$ in [7] using the ENO2 scheme. A comparison of perfect flow (dash-line) and equilibrium flow (solid-line). (a) and (b) At the left solid endwall; (c) and (d) at the right solid endwall. 
It corresponds to the test case $\mathrm{C}$ applied by Montagné et al. [7]. This case is a difficult test for all schemes, either for the perfect or real gases examined in [7]. There are noticeable errors on the levels of the velocities and on the position of the shock, all schemes have problems at the contact discontinuity for the real gas [7]. The initial cell size are the same as the previous example. The properties of pressure and temperature computed with both first-order Godunov and second-order ENO schemes are shown along with the exact solution in Fig. 14 at time $t=0.3 \mathrm{~ms}$. Good continuities for the pressure and local flow velocity in contact discontinuity can be obtained. Our numerical results agree well with the exact solutions and the ENO2 results indicate better resolution at shock and at the rarefaction wave. Other flow properties computed with ENO2 scheme are also shown along with the exact solution in Fig. 15 at time $t=0.3 \mathrm{~ms}$. Very good agreement is found between the computation and exact solution. The influence of real gas effect in the moving normal shock and the

(a)

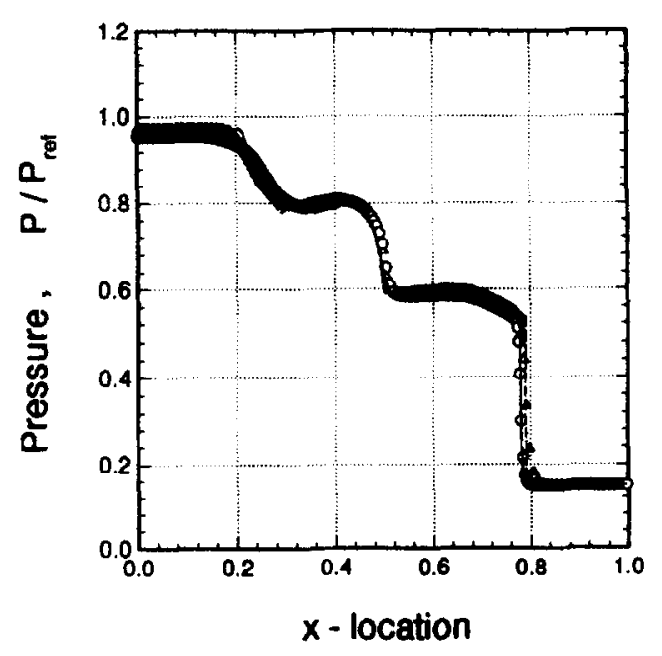

(c)

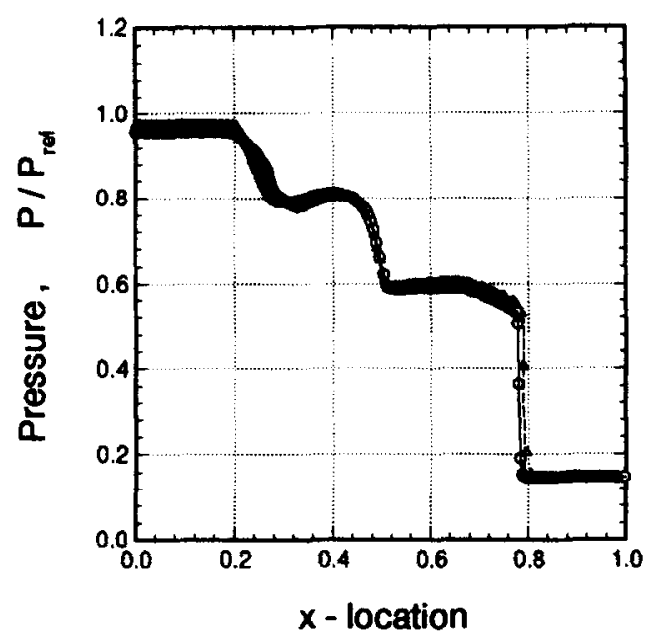

(b)

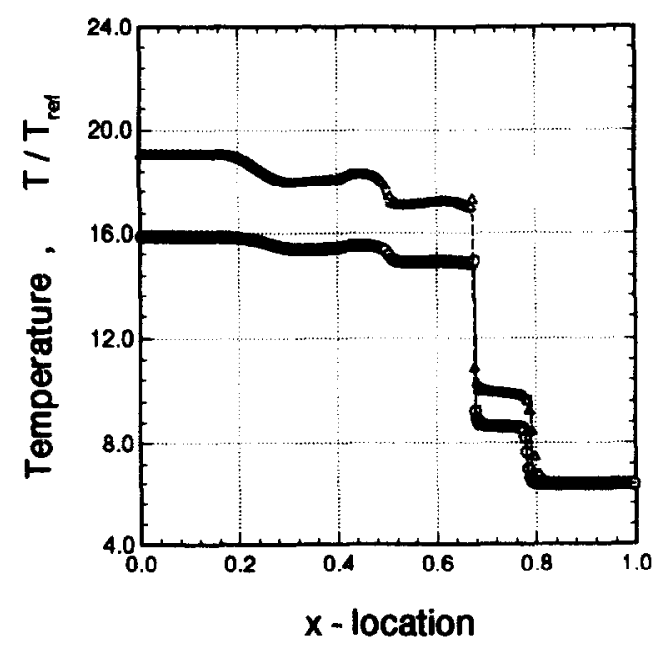

(d)

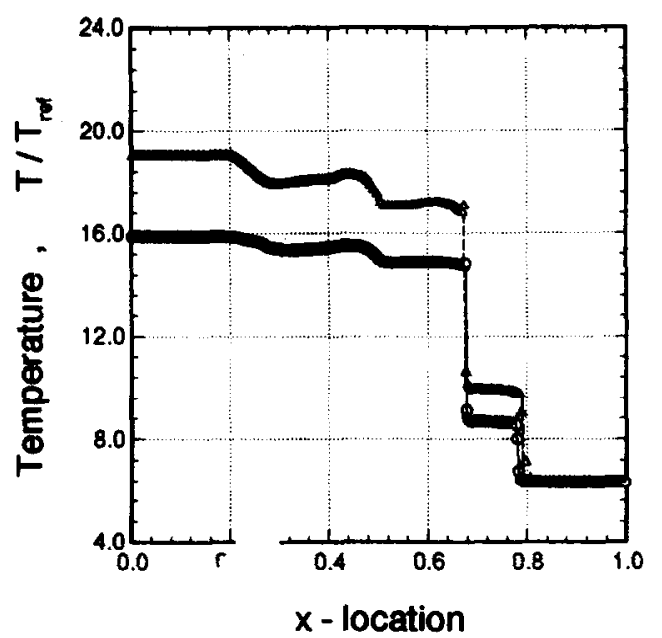

Fig. 19. Flow properties of a high-temperature shock tube flow for test case $A$ in [7] with a conical flare using the ENO2 scheme at time $t=0.2 \mathrm{~ms}$. A comparison of numerical results for perfect air (triangles) and equilibrium air (circles) and exact solution for perfect air (dash-line) and equilibrium air (solid-line). (a) Pressure; (b) temperature; (c) density; (d) velocity. 
thermodynamic properties behind the moving shock is the same as the above case. Notice that although the ratio of specific heats, $\gamma=\rho a^{2} / P$, equal to 1.4 and $1.16 \sim 1.17$, respectively, as seen in Fig. 15(a), no wiggle occurs for our pathline Godunov scheme. The computed pathline grids and the time history of pressure-contour distribution for the perfect and equilibrium air flows using ENO2 scheme are presented in Figs. 16 and 17. Flowfield structures which follow the movement of the fluid particles are clearly demonstrated. The time history of the surface flow properties at the left and right solid endwalls for perfect and equilibrium air gases are also shown in Fig. 18. From Fig. 18, we can conclude that this case is a good shock-tube condition for the design of the reflection shock tunnel due to an attainment of good flow conditions in a long time duration.

To evaluate the robustness of the splitting technique for the system Eq. (1) involving source terms in our pathline Godunov method, we consider here a shock tube with variable area distribution for the flow conditions and the initial cell sizes the same as the above two high-temperature cascs. The geometry of variable-area shock tube consists of a high pressure driver section of $0.4 \mathrm{~m}$ long and a low pressure driven section of $0.5 \mathrm{~m}$ long, and are connected with a same high pressure conical flare section of $0.1 \mathrm{~m}$ long between them. The area ratio between high and low pressure section is equal to 4 , i.e. $A_{\mathrm{L}} / A_{\mathrm{R}}=4$. Note that the exact solutions for the following two cases are obtained with fine grids of 2500 cells at high pressure side and 500 cells at low pressure side with different initial cell sizes $\Delta x_{\mathrm{ini}}=0.0002$ and 0.001 , respectively.

Firstly, the flow properties for the test case A in [7] computed with ENO2 scheme along with the exact solution are shown in Fig. 19 at time $t=0.2 \mathrm{~ms}$. All numerical results agree well with the exact solutions. The

(a) Perfect Air

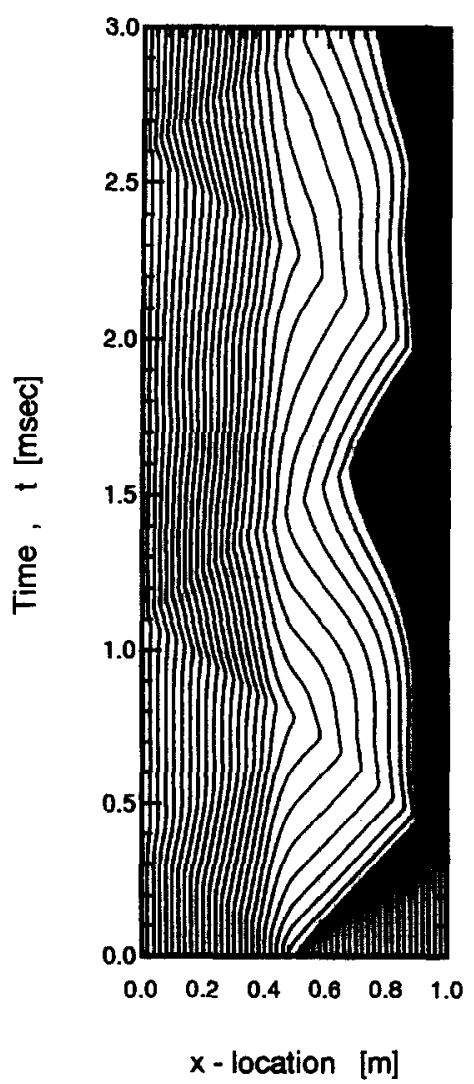

(b) Equilibrium Air

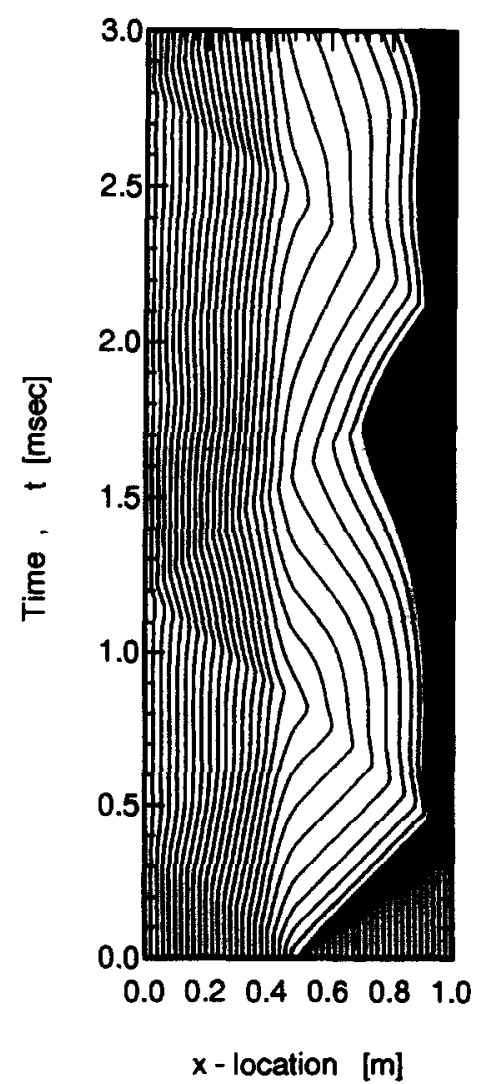

Fig. 20. Pathline grids of a high-temperature shock tube flow for test case A in [7] with a conical flare using the ENO2 scheme. (a) Perfect air flow; (b) equilibrium air flow. 
computed pathline grids and the time history of temperature-contour distribution for the perfect and equilibrium air flows using ENO2 scheme are presented in Figs. 20 and 21, respectively. Compared with the constant area case, flowfield structures are more complicated, especially at the section of conical flare. The time history of the surface flow properties at the left and right solid endwalls for perfect and equilibrium air gases are also shown in Fig. 22. The same effect of the existence of conical flare on the surface flow properties can also be seen. Compared with the constant area case, the values of total enthalpy $h_{0}$ at the left and right solid endwalls experience a large change with the existence of conical flare, for both the perfect and equilibrium air gas, as shown in Fig. 23.

For the test case $\mathrm{C}$ in [7], the computed flow properties with ENO2 scheme along with the exact solution are shown in Fig. 24 at time $t=0.3 \mathrm{~ms}$. Again, all numerical results agree well with the exact solutions. The computed pathline grids and the time history of pressure-contour distribution for the perfect and equilibrium air flows using ENO2 scheme are presented in Figs. 25 and 26. The time history of the surface flow properties at the left and right solid endwalls for perfect and equilibrium air gases are also shown in Fig. 27. The influence on the flowfield structure and the time history of the surface flow properties due to the presence of conical flare is evident. In Fig. 28, the values of total enthalpy $h_{0}$ at the left and right solid endwalls experience a great increase due to the existence of conical flare for both the perfect and equilibrium gases. As shown in Fig. 28, with a higher total enthalpy $h_{0, e q}$ and a longer time duration of steady flow conditions at the right solid endwall, it indicates that the application of the conical flare is useful for the design of the reflection shock tunnel.

(a) Perfect Air

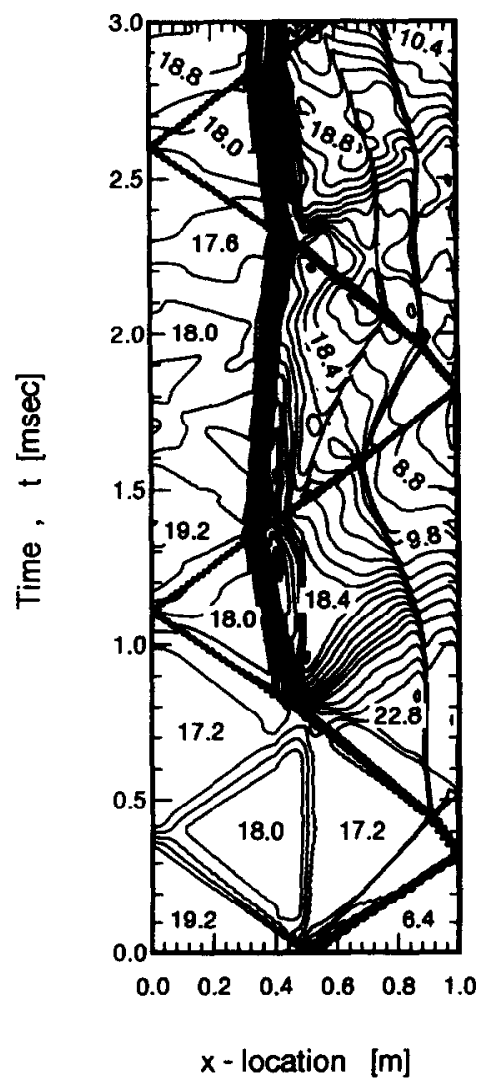

(b) Equilibrium Air

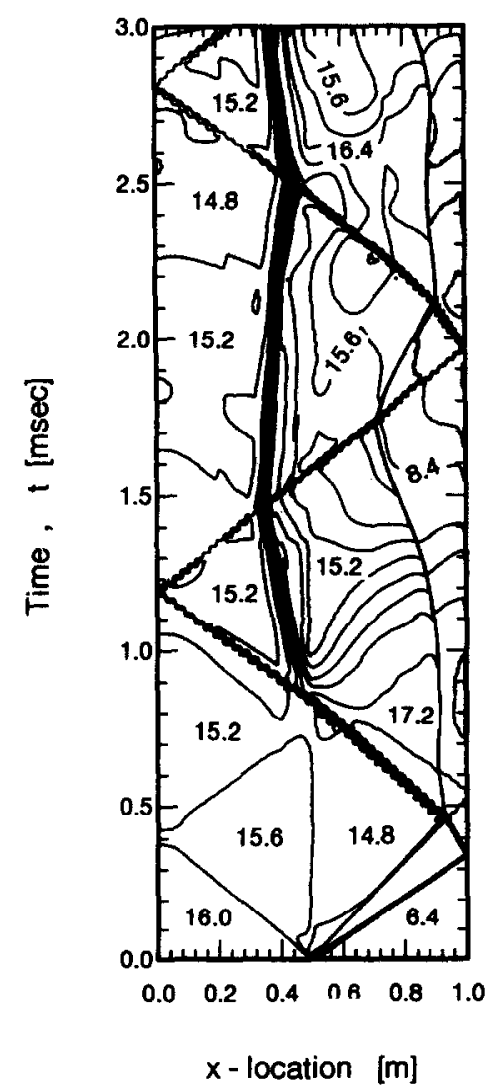

Fig. 21. Time history of temperature-contour distribution of a high-temperature shock tube flow for test case A in [7] with a conical flare using the ENO2 scheme. (a) Perfect air flow; (b) equilibrium air flow. 
(a)

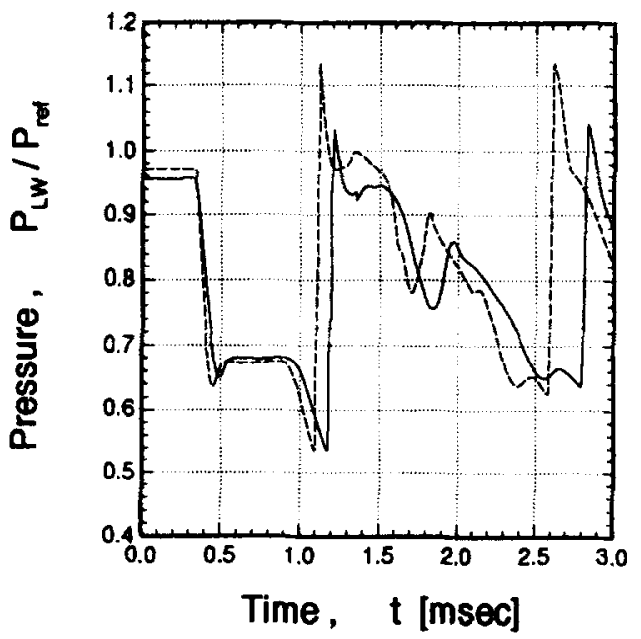

(c)

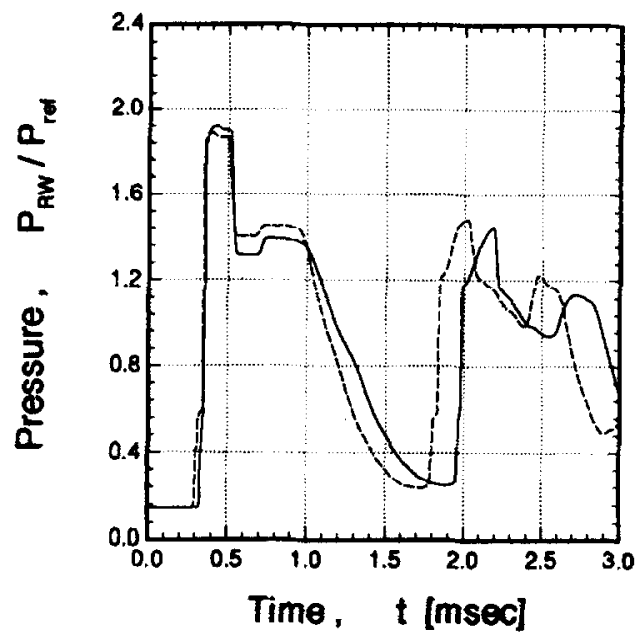

(b)

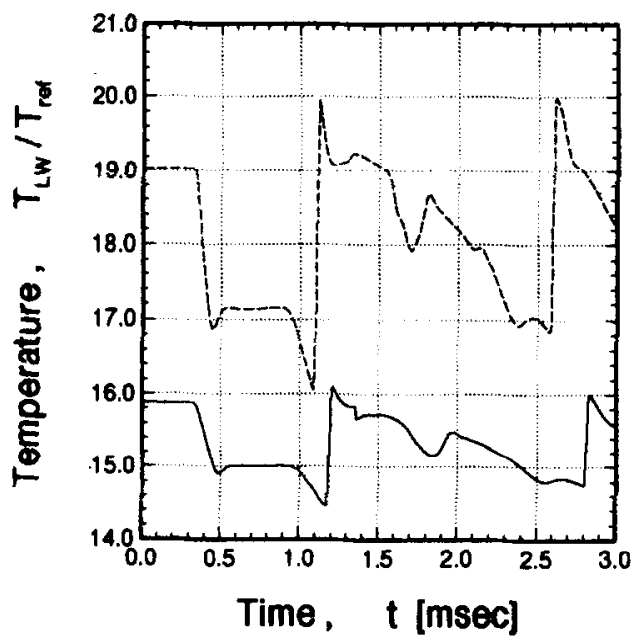

(d)

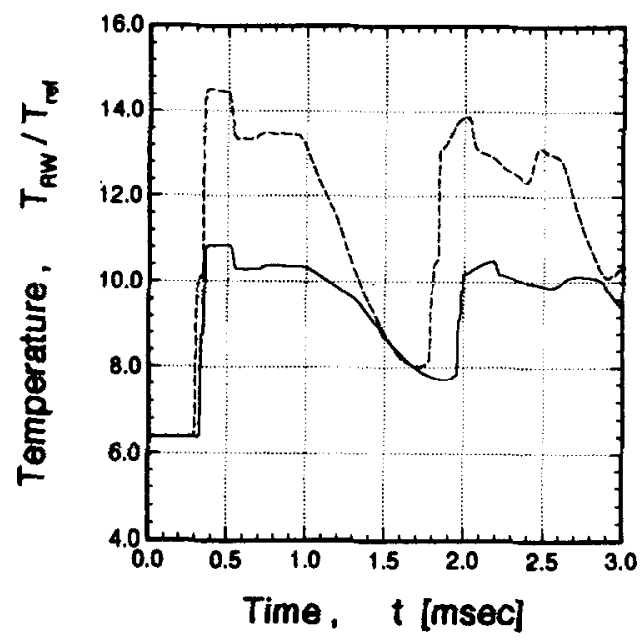

Fig. 22. Time history of surface flow properties at the left and right solid endwalls for test case A in [7] with a conical flare using the ENO2 scheme. A comparison of perfect flow (dash-line) and equilibrium flow (solid-line). (a) and (b) At the left solid endwall; (c) and (d) at the right solid endwall. 
(a) Perfect Air Flow

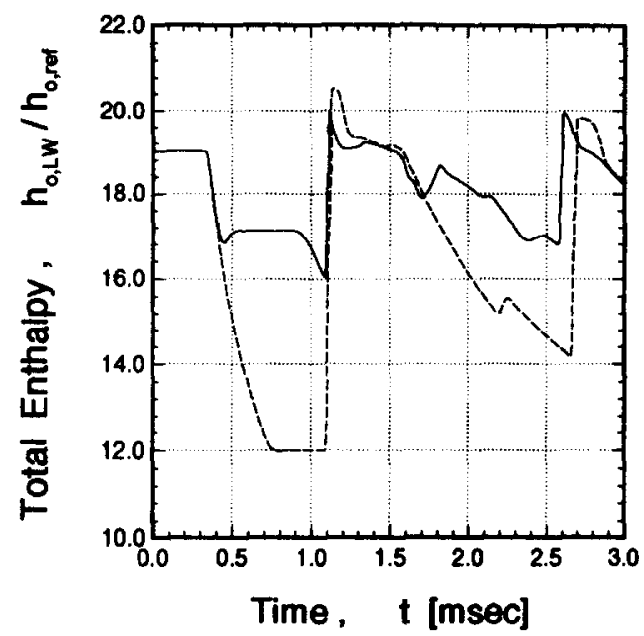

(c) Perfect Air Flow

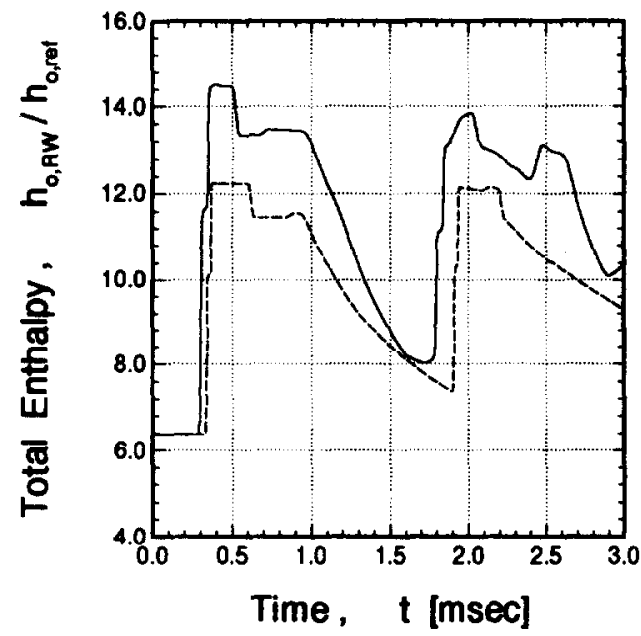

(b) Equilibrium Air Flow

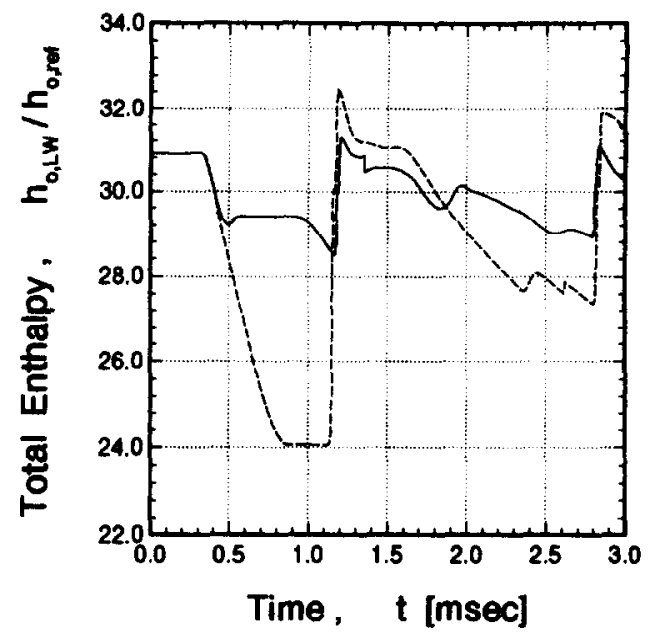

(d) Equilibrium Air Flow

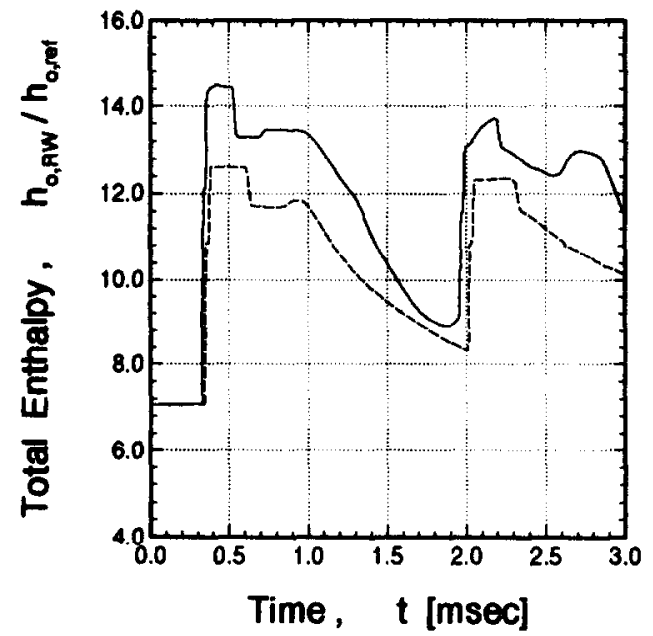

Fig. 23. The effect of existence of conical flare in the time history of the total enthalpy $h_{0}$ for test case $\mathrm{A}$ in [7] at the left and right solid endwalls using the ENO2 scheme. A comparison of constant area case (dash-line) and case with conical flare (solid-line). (a) and (b) At the left solid endwall; (c) and (d) at the right solid endwall. 
(a)

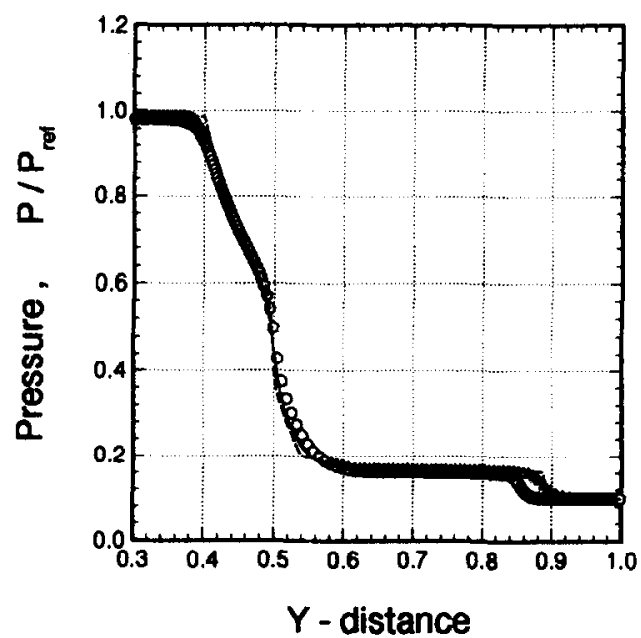

(c)

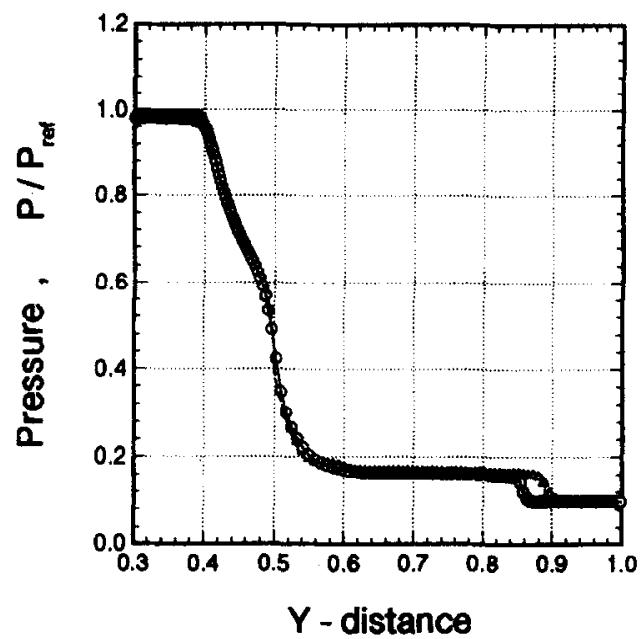

(b)

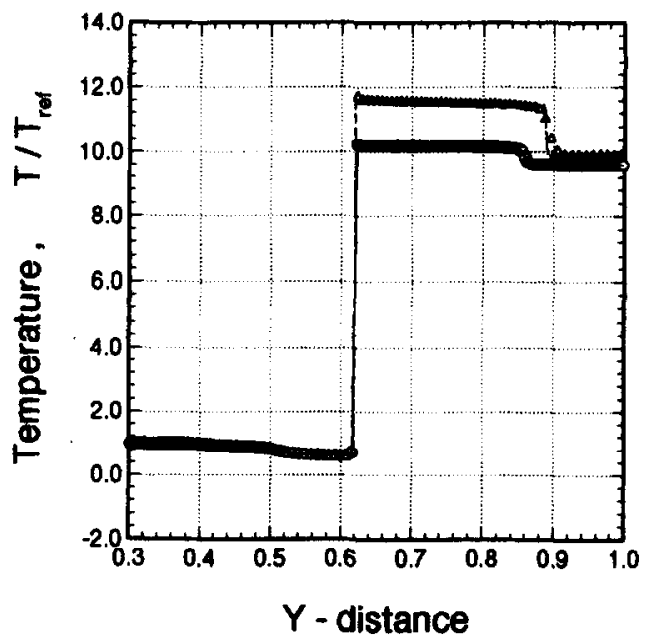

(d)

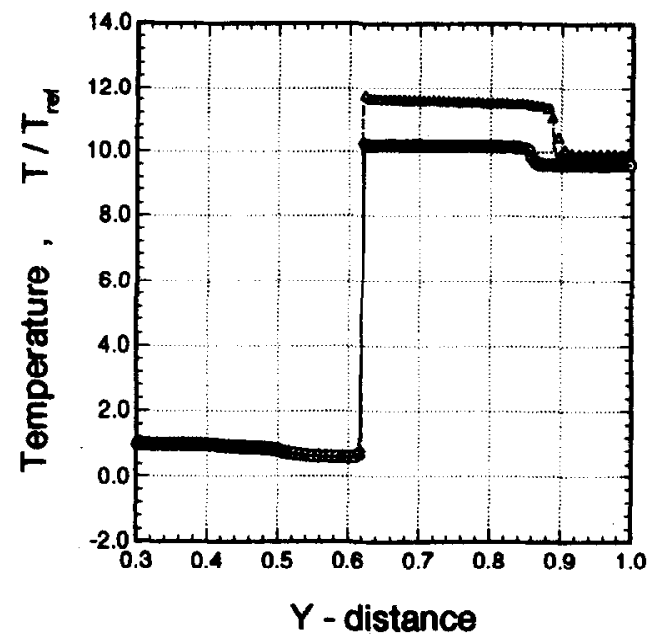

Fig. 24. Flow properties of a high-temperature shock tube flow for test case $\mathrm{C}$ in [7] with a conical flare using the ENO2 scheme at time $t=0.3 \mathrm{~ms}$. A comparison of numerical results for perfect air (triangles) and equilibrium air (circles) and exact solution for perfect air (dash-line) and equilibrium air (solid-line). (a) Pressure; (b) temperature; (c) density; (d) velocity. 
(a) Perfect Air

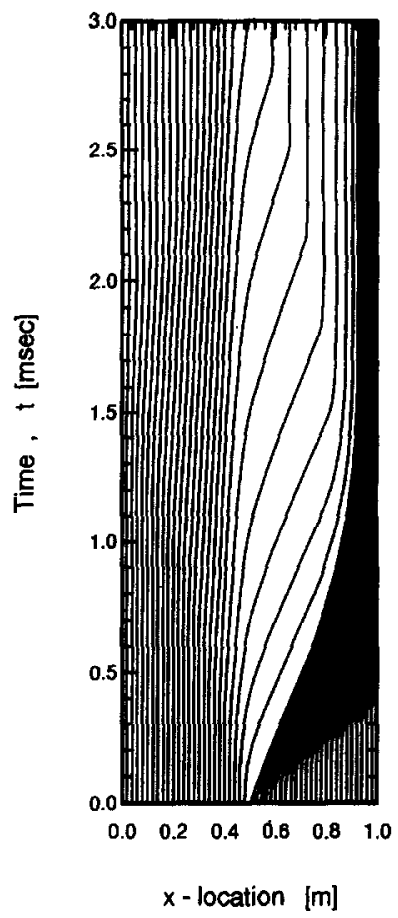

(b) Equilibrium Air

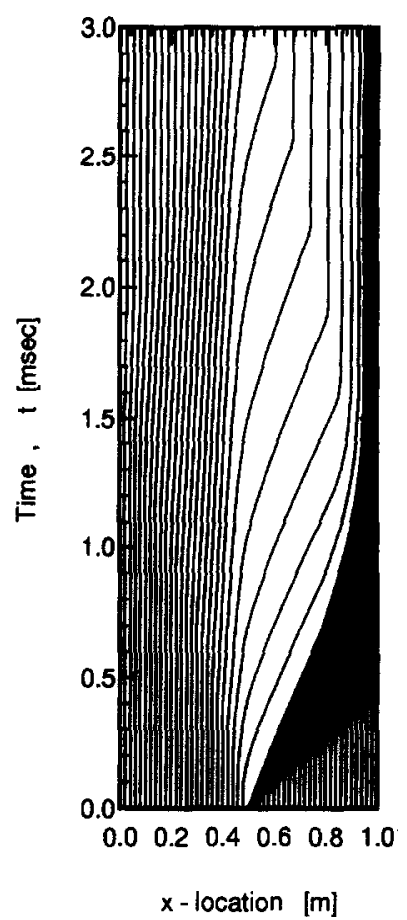

Fig. 25. Pathline grids of a high-temperature shock tube flow for test case $\mathrm{C}$ in [7] with a conical flare using the ENO2 scheme. (a) Perfect air flow; (b) equilibrium air flow.

(a) Perfect Air

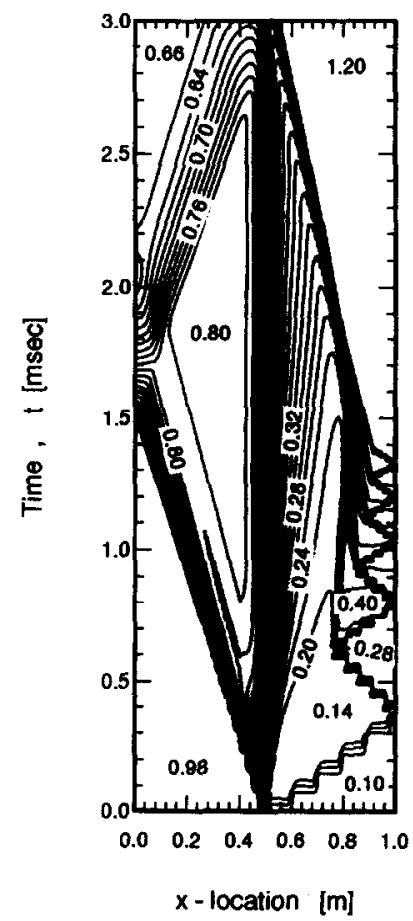

(b) Equilibrium Air

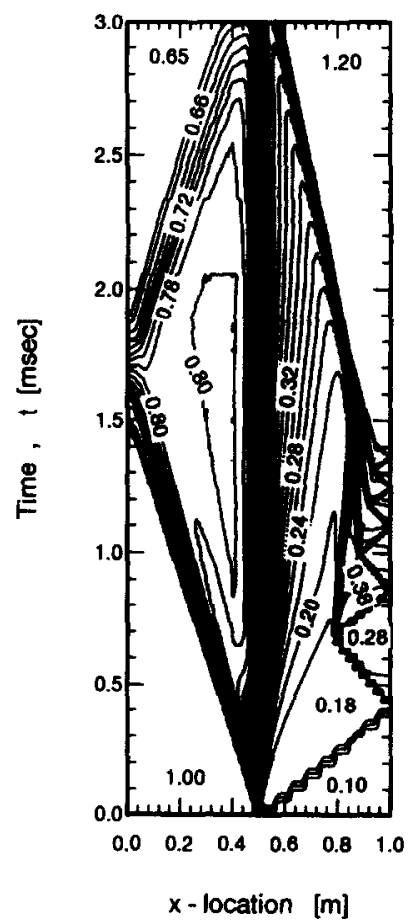

Fig. 26. Time history of temperature-contour distribution of a high-temperature shock tube flow for test case $\mathrm{C}$ in [7] with a conical flare using the ENO2 scheme. (a) Perfect air flow; (b) equilibrium air flow. 
(a)

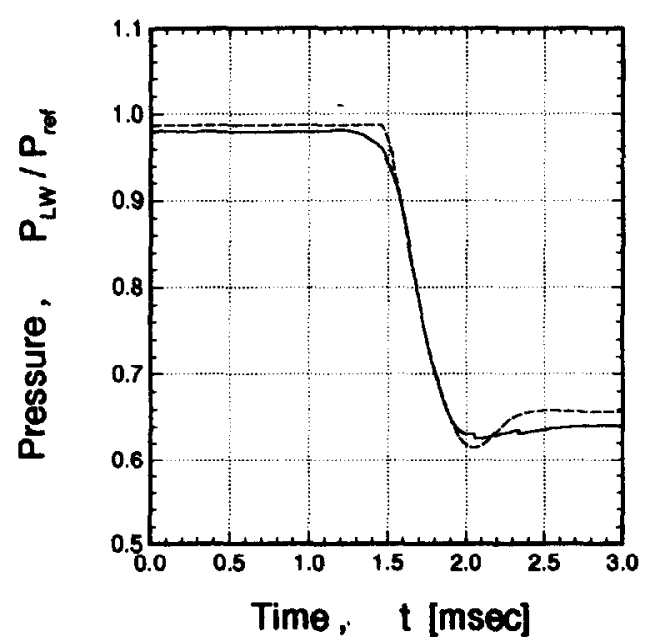

(c)

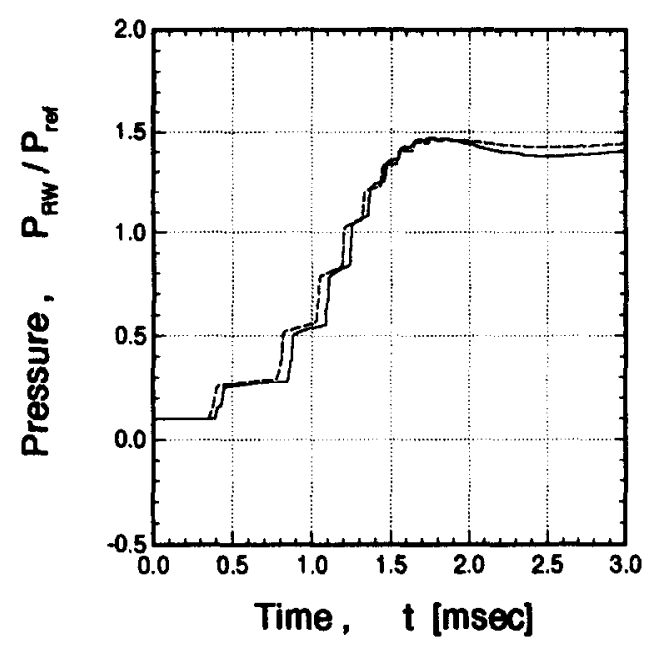

(b)

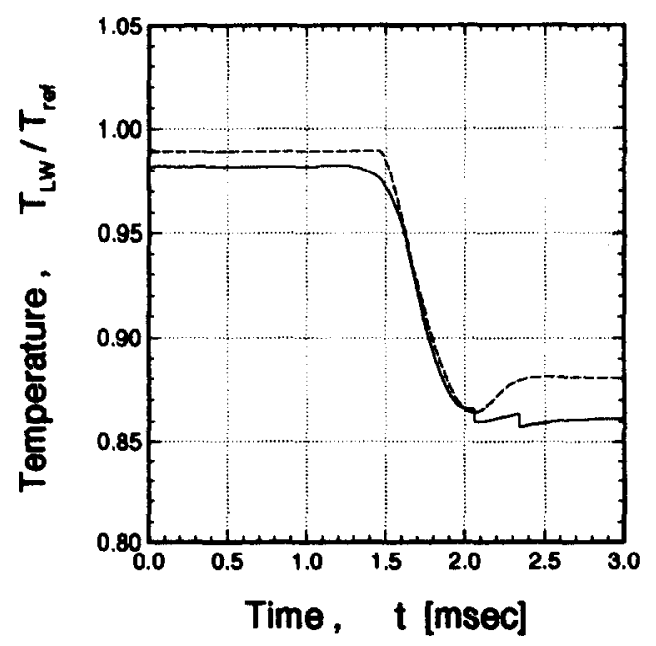

(d)

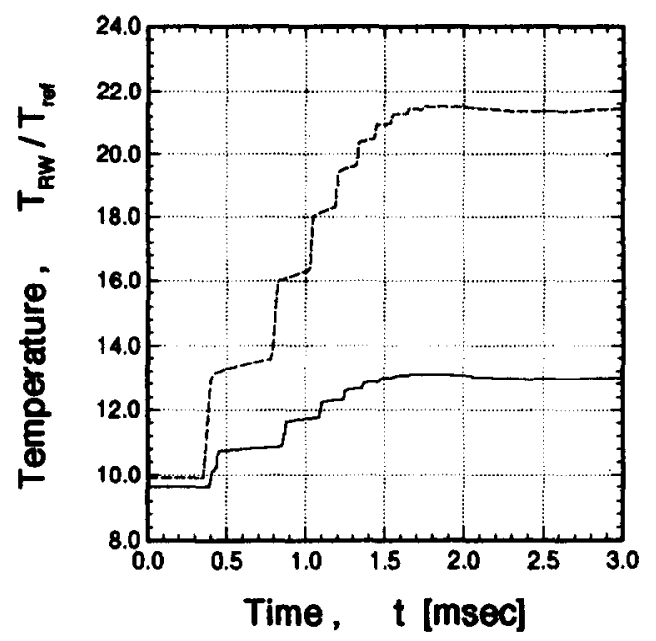

Fig. 27. Time history of surface flow properties at the left and right solid endwalls for test case $\mathrm{C}$ in [7] with a conical flare using the ENO2 scheme. A comparison of perfect flow (dash-line) and equilibrium flow (solid-line). (a) and (b) At the left solid endwall; (c) and (d) at the right solid endwall. 
(a) Perfect Air Flow

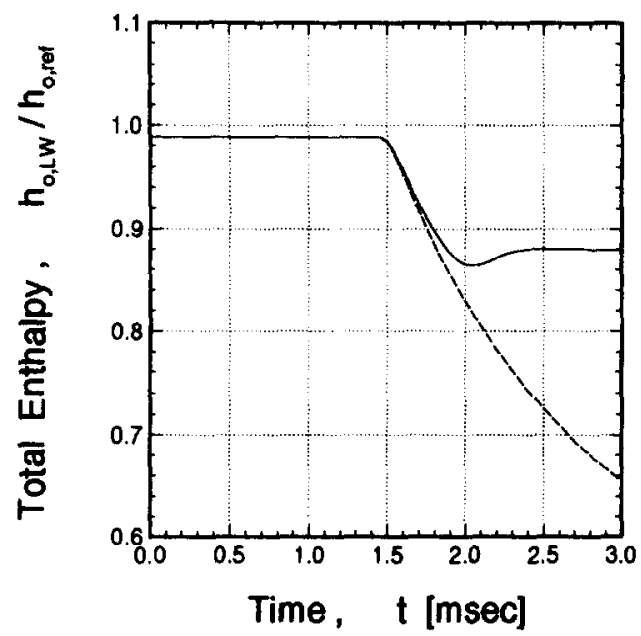

(c) Perfect Air Flow

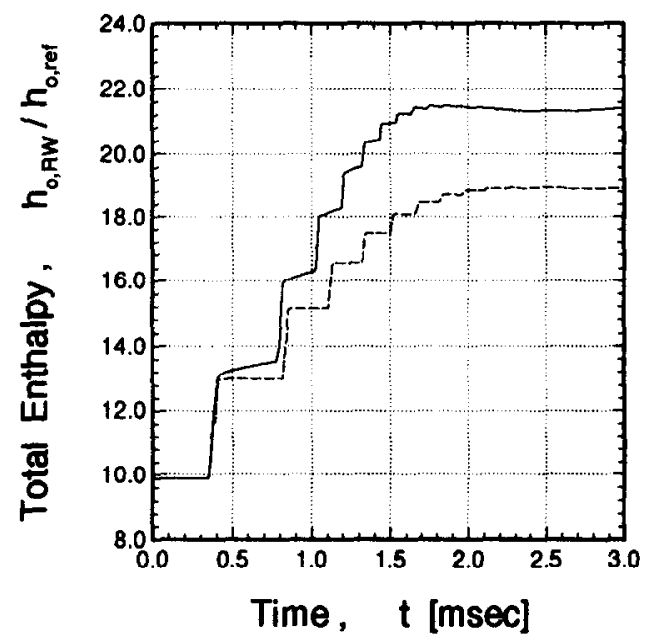

(b) Equilibrium Air Flow

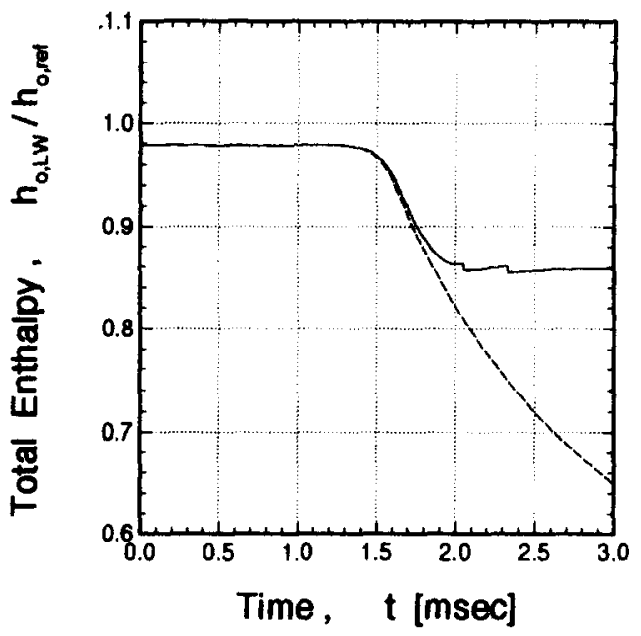

(d) Equilibrium Air Flow

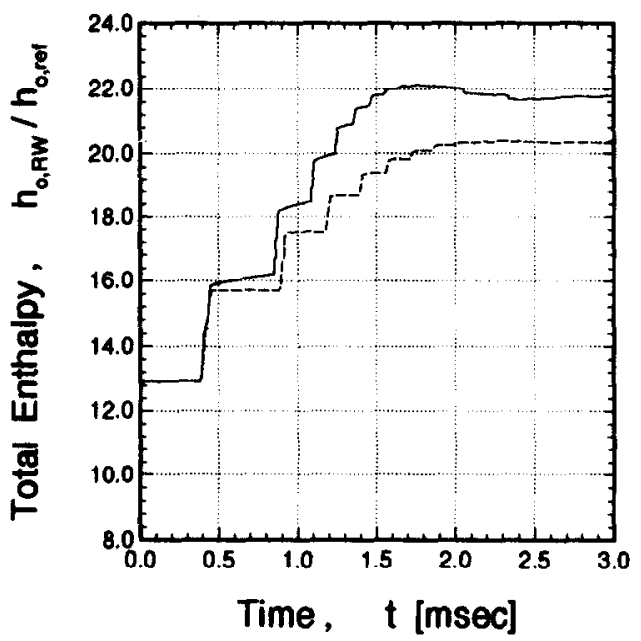

Fig. 28. The effect of existence of conical flare in the time history of the total enthalpy $h_{0}$ for test case $\mathrm{C}$ in [7] at the left and right solid endwalls using the ENO2 scheme. A comparison of constant area case (dash-line) and case with conical flare (solid-line). (a) and (b) At the left solid endwall; (c) and (d) at the right solid endwall. 


\section{Concluding remarks}

In this paper, a Godunov-type method with pathline meshes as control cells is presented for solving the unsteady quasi-one-dimensional equilibrium flows. The equilibrium real gas Riemann problem and its solution in the pathline mesh system is devised for the implementation of Godunov method. Tannehill's real gas model is adopted to calculate the equilibrium gas properties. A two-step essentially non-oscillatory method is implemented to achieve high-order accuracy. Computations of unsteady quasi-one-dimensional equilibrium flows have been carried out and the results indicate that the smooth flow can be accurately represented and flow discontinuities such as shock waves and contact discontinuities can be crisply resolved. The use of pathline meshes as computational cells enables intrinsic flow adaption, excellent resolution of contact discontinuities, and simpler implementation of the Godunov method. The computational algorithm is robust and accurate and is very suitable for unsteady quasi-one-dimensional equilibrium flow calculations. Application to the simulation of flow in a high-temperature shock tube with conical flare indicates that the present method can be useful for the analysis and design of a reflection shock tunnel. Extension of the present pathline Godunov-type scheme to unsteady quasi-one-dimensional nonequilibrium flows is a subject of future study.

\section{Acknowledgment}

This work was sponsored by the National Science Council of the Republic of China under Grant NSC 86-2612E002-010 and NCHC 86-04-003.

\section{References}

[1] C.A. Hsu and J.Y. Yang, A high-order streamline Godunov scheme for steady supersonic flow computation, J. Comput. Methods Appl. Mech. Engrg. 124 (1995) 283-302.

[2] J.Y. Yang, Y.H. Tang and S.T. Lee, A high-order streamline Godunov scheme for steady supersonic/hypersonic equilibrium flows, Comput. Methods Appl. Mech. Engrg. 159 (1998) 261-289.

[3] S.K. Godunov, A difference method for numerical calculation of discontinuous solutions in hydrodynamics, Mathematicheskii Sbornik 47 (1959) 271-306.

[4] P. Colella and H.M. Glaz, Efficient solution algorithms for the Riemann problem of real gases, J. Comput. Phys. 59 (1985) $264-289$.

[5] P. Glaister, An approximate linearised Riemann solver for Euler equations for real gases, J. Comput. Phys. 74 (1988) $382-408$.

[6] B. Grossman and R.W. Walters, Analysis of flux-split algorithms for Euler's equations with real gases, AIAA J. 27(5) (1989) 524-531.

[7] J.L. Montagné, H.C. Yee and M. Vinokur, Comparative study of high-resolution shock-capturing schemes for a real gas, AIAA J. 27(10) (1989) 1332-1346.

[8] A. Suresh and M.S. Liou, Osher's scheme for real gases, AIAA J. 29(6) (1991) 920-926.

[9] M. Vinokur and J.L. Montagné, Generalized flux-vector splitting and Roe average for an equilibrium real gas, J. Comput. Phys. 89 (1990) 276-300.

[10] S. Srinivasan, J.C. Tannehill and K.J. Weilmunster, Simplified curve fit for the thermodynamic properties of equilibrium air, NASA Reference Publication 1181, August 1987.

[11] J.L. Steger and R.F. Warming, Flux vector splitting of inviscid gasdynamic equations with application to finite difference methods, J. Comput. Phys. 40 (1981) 263-293.

[12] B. Van Leer, Flux-vector splitting for the Euler equations, ICASE Report 82-30, September 1982.

[13] P.L. Roe, Approximate Riemann solvers, parameter vectors, and difference schemes, J. Comput. Phys. 43 (1981) 357-372.

[14] M. Vinokur and Y. Liu, Equilibrium gas flow computation II. An analysis of numerical formulations of conservation laws, AIAA Paper 88-0127, January 1988.

[15] C.Y. Loh and W.H. Hui, A new Lagrangian method for steady supersonic flow computation. I. Godunov scheme, J. Comput. Phys. 89 (1990) 207-240.

[16] A. Harten and S. Osher, Uniformly second-order essentially nonoscillatory schemes, I, SIAM J. Numer. Anal. 24(2) (1987) $279-309$.

[17] G.A. Sod, A survey of several finite difference methods of systems of nonlinear hyperbolic conservation laws, J. Comput. Phys. 27 (1978) $1-31$.

[18] S. Karni, Multi-component flow calculations by a consistent primitive algorithm, J. Comput. Phys. 112 (1994) $31-43$. 